\title{
Meander dynamics: A nonlinear model without curvature restrictions for flow in open-channel bends
}

\author{
K. Blanckaert ${ }^{1,2,3}$ and H. J. de Vriend ${ }^{2}$ \\ Received 5 March 2009; revised 5 November 2009; accepted 29 April 2010; published 20 October 2010.
}

[1] Despite the rapid evolution of computational power, simulation of meander dynamics by means of reduced and computationally less expensive models remains practically relevant for investigation of large-scale and long-term processes, probabilistic predictions, or rapid assessments. Existing meander models are invariantly based on the assumptions of mild curvature and slow curvature variations and fail to explain processes in the high-curvature range. This article proposes a nonlinear model for meander hydrodynamics without curvature restrictions. It provides the distribution of the main flow, the magnitude of the secondary flow, the direction of the bed shear stress, and the curvature-induced additional energy losses. It encompasses existing mild curvature models, remains valid for straight flow, and agrees satisfactorily with experimental data from laboratory experiments under conditions that are more demanding than sharp natural river bends. The proposed model reveals the mechanisms that drive the velocity redistribution in meander bends and their dependence on the river's roughness $C_{\mathrm{f}}$, the flow depth $H$, the radius of curvature $R$, the width $B$, and bathymetric variations. It identifies $C_{\mathrm{f}}^{-1} H / R$ as the major control parameter for meander hydrodynamics in general and the relative curvature $R / B$ for sharp curvature effects. Both parameters are small in mildly curved bends but $O(1)$ in sharply curved bends, resulting in significant differences in the flow dynamics. Streamwise curvature variations are negligible in mildly curved bends, but they are the major mechanisms for velocity redistribution in sharp bends. Nonlinear feedback between the main and secondary flow also plays a dominant role in sharp bends: it increases energy losses and reduces the secondary flow, the transverse bed slope, and the velocity redistribution.

Citation: Blanckaert, K., and H. J. de Vriend (2010), Meander dynamics: A nonlinear model without curvature restrictions for flow in open-channel bends, J. Geophys. Res., 115, F04011, doi:10.1029/2009JF001301.

\section{Introduction}

[2] Meandering continues to interest scientists, practitioners, and public administrations. In many parts of the world, people tend to concentrate in low-lying areas, causing an ongoing encroachment of lowland rivers by human activities in the riparian zones. This makes bank erosion and meander migration ever less desirable. The depletion of the world's hydrocarbon stocks increases the pressure on geologists to find the new reserves [Swanson, 1993]. This creates a need for more detailed insight into how deposits have been formed at geological timescales. Both examples illustrate the need for simulation, hindcast, and prediction capabilities, i.e., in the former case concerning the probability of bank erosion at

\footnotetext{
${ }^{1}$ LCH-ENAC, Ecole Polytechnique Fédérale Lausanne, Lausanne, Switzerland.

${ }^{2}$ Department of Civil Engineering and Geosciences, Delft University of Technology, Delft, The Netherlands.

${ }^{3}$ State Key Laboratory of Urban and Regional Ecology, Research Center for Eco-Environmental Sciences, Chinese Academy of Sciences, Beijing, China.

Copyright 2010 by the American Geophysical Union. 0148-0227/10/2009JF001301
}

short and intermediate timescales and in the latter case concerning the formation of meander deposits at geological timescales. Although significant progress has been made in recent years, there are still important knowledge gaps in both areas.

[3] The rapid evolution in computational power has allowed ever more complicated and process-based numerical modeling. Three-dimensional numerical modeling of flow and morphology in laboratory flumes started in 1990 [Shimizu et al., 1990; Wu et al., 2000; Minh Duc et al., 2004; Ruther and Olsen, 2005; Khosronejad et al., 2007; Zeng et al., 2008], and nowadays, simulation of real river configurations becomes feasible [Fischer-Antze et al., 2008]. Meander evolution has been simulated with a two-dimensional model by Duan et al. [2001] and, recently, with a three-dimensional model by Ruther and Olsen [2007] on a laboratory scale.

[4] Despite the rapid evolution in computational power, simulation of meandering river flow and morphology by means of reduced and computationally less expensive models is still practically relevant not only for rapid assessments but also for probabilistic morphological predictions [e.g., van Vuren et al., 2005] and basin-scale simulations of morphology and sediment deposit structures [Sun et al., 2001; Clevis et al., 2006a, 2006b; Cojan et al., 2005]. One may also claim 
that such reduced models are in some respects simpler and more transparent, hence more insightful. On the other hand, this simplification has, of course, its price in terms of a less generic model concept.

[5] Meander models typically consist of three components that describe (1) the meander planform and migration, (2) the meander morphology, and (3) the hydrodynamics. The three components are intrinsically coupled because the morphology and the sediment transport are basically driven by their interaction with the hydrodynamics. Obviously, simplified meander models only intend to describe the global macroscopic features of the planform, the morphology, and the flow field and do not intend to resolve small-scale or short-term features such as migrating bed forms, details of the timeaveraged flow patterns, or turbulent structures. The present article focuses on the hydrodynamic component. Existing meander models are invariantly based on the assumption of mild curvature and slow curvature variations but are commonly applied to investigate meander dynamics in the entire curvature range [Howard, 1984; Liverpool and Edwards, 1995; Stølum, 1996, 1998; Lancaster and Bras, 2002; Sun et al., 2001; Edwards and Smith, 2002; Camporeale et al., $2005,2008]$. They are able to reproduce characteristic meander features in simulations of large-scale and long-term meander dynamics that look remarkably realistic but may nevertheless be fundamentally flawed because of the neglect of high-curvature effects. Although these models allowed significant progress in unraveling and understanding meander dynamics [Seminara, 2006], they fail to clarify meander processes at high curvature such as the conditions of occurrence of cutoffs of meander bends and the formation of oxbow lakes, which are known to play a fundamental role in the dynamic evolution of the meander planform and the floodplain stratigraphy [Sun et al., 2001; Clevis et al., 2006a, 2006b; Liverpool and Edwards, 1995; Edwards and Smith, 2002; Camporeale et al., 2008].

[6] The major objective of the present article is to propose a model for the horizontal distribution of the flow, which, together with the model for the vertical structure of the flow [Blanckaert and de Vriend, 2003] and the model for the turbulent kinetic energy [Blanckaert, 2009], forms a fully nonlinear hydrodynamic model that is valid for openchannel bends irrespective of the planform and curvature, including the asymptotic case of straight flow, encompasses existing models limited to mild curvature, and is computationally hardly more expensive.

[7] Section 2 schematizes the components of meander models, briefly describes their interactions, reviews existing models, and situates the contribution and originality of the present article. The hydrodynamic model without curvature restrictions is derived in section 3, validated in section 4 by means of theoretical asymptotic cases and laboratory experiments, and analyzed in section 5. The application of the model to investigate meander dynamics will be reported elsewhere.

\section{Outline and Contribution of the Proposed Model}

2.1. Outline of the Proposed Meander Model and Interaction among Physical Processes

[8] Camporeale et al. [2007, Figure 2] have described in detail the interrelationships among the physical processes that act in meandering rivers. The contribution of the present article concerns the hydrodynamic component of meander models. The outline of the entire meander model will now briefly be presented, however, to contextualize the contribution of the hydrodynamic model and to illustrate the implementation of the interrelationships among the physical processes. Figure 1 illustrates these interrelationships within the mathematical formulation of the proposed model.

[9] This article adopts a right-handed reference system $(s, n, z)$ (Figure 1), where the vertical $z$ axis points upward and the curvilinear $s$ axis follows the river centerline in streamwise direction. Cross sections are oriented perpendicular to the centerline along the $n$ axis, which is positive toward the left. As a consequence, meander bends turning to the left/right are characterized by a negative/positive centerline radius of curvature $R$. A metric factor $1+n / R$ accounts for different streamwise distances along the centerline and at the transverse position $n$.

[10] On the (generally satisfied) condition that the crosssectional averaged flow depth and velocity vary in streamwise direction on a much larger spatial scale than the local flow depth and velocity, the meander model can be decomposed in the following interacting submodels, as illustrated in Figure 1.

\subsubsection{A Large-scale One-dimensional Morphodynamic} Submodel

[11] This submodel, which describes processes on the scale of the river basin, computes cross-sectional averaged values of the bed elevation $Z_{\mathrm{b}}$, the water surface elevation $Z_{\mathrm{s}}$, the flow depth $H$ equal to $Z_{\mathrm{s}}-Z_{\mathrm{b}}$, and the velocity $U$ (Figure 1 ). It allows accounting for backwater effect, such as those due to variations in sea level. This submodel is affected by the changes of the meander length because of planform changes as well as the curvature-induced additional energy losses, which account for the interaction with the other submodels.

\subsubsection{A Meander Migration Submodel}

[12] The meander migration submodel determines the meander planform. Hence, it provides the centerline radius of curvature $R$, which parameterizes the curvature effects in the meander model. Meander migration occurs at time scales that are typically at least an order of magnitude larger than morphologic and hydrodynamic changes, which allow a decoupled treatment, i.e., computing the morphologic and hydrodynamic characteristics in steady planforms.

[13] Meander migration occurs through erosion and bank retreat at the outer bank and accretion and bank advance at the inner bank. Because meanders are characterized by a constant width $B$, at least from a statistical and long-term point of view, meander models assume the rate of bank accretion to be equal to the rate of bank erosion. The meander migration rate $M$ is commonly expressed in meander models as a function of the velocity excess $\Delta U_{\mathrm{s}}$ and the flow-depth excess near the outer bank $\Delta h$, which are defined as the difference between their maximum values near the outer bank and their cross-sectional averaged values (Figure 1):

$$
M=f c t\left(\Delta U_{\mathrm{s}}, \Delta h\right) .
$$

[14] Although this simple model is justified by field observations [Pizzuto and Meckelnburg, 1989], it accounts neither for the geotechnical processes and external factors such 


\section{MEANDER MODEL}
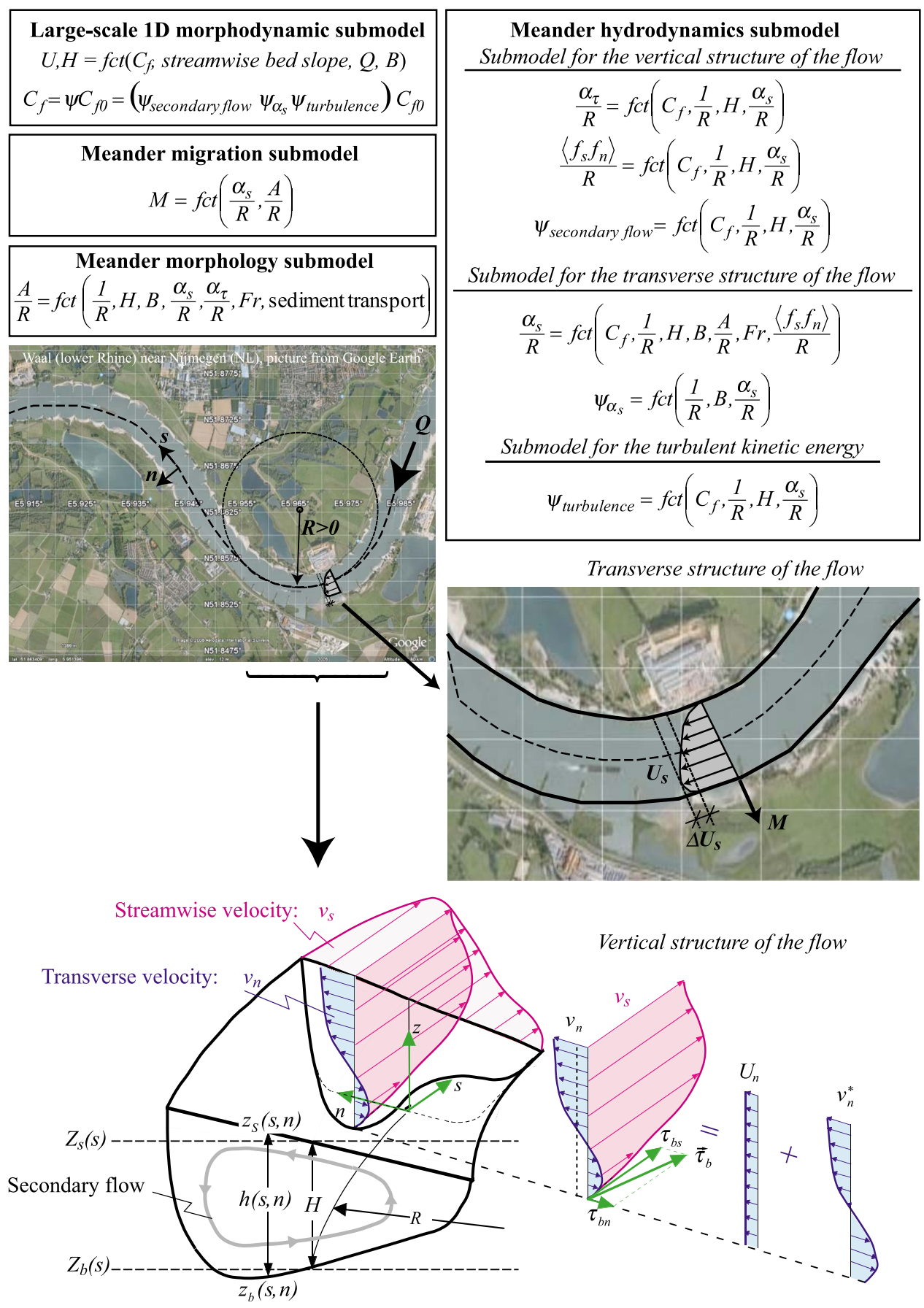

Datum

Figure 1. Concept of the meander model and schematization of interdependencies between different submodels and variables within the mathematical formulation of the proposed model; definition of reference system and notations.

as groundwater flow and ship waves nor for the complex near bank hydrodynamics, such as the outer bank cell of secondary flow [e.g., Mockmore, 1943; Bathurst et al., 1979; Blanckaert and de Vriend, 2004], the flow recirculation at the inner bank [Leeder and Bridges, 1975; Ferguson et al., 2003; Blanckaert, 2010], or the effects of bed form progression [Abad and Garcia, 2009], which are often observed in open-channel bends and may be relevant for meander migration.

\subsubsection{A Meander Morphology Submodel}

[15] Because the large-scale one-dimensional morphodynamic submodel provides the cross-sectional averaged bed 
elevation $Z_{\mathrm{b}}$, the meander morphology submodel has to provide the transverse profile of the riverbed $z_{\mathrm{b}}$, including the flow-depth excess near the outer bank $\Delta h$. The transverse bed slope is determined by the direction of the sediment transport $q_{\mathrm{bn}} / q_{\mathrm{bs}}$, the downslope gravitational pull on the sediment particles $-G \partial z_{\mathrm{b}} / \partial n$, the direction of the depthaveraged velocity $U_{\mathrm{n}} / U_{\mathrm{s}}$, and the upslope drag force on the sediment particles exerted by the transverse component of the bed shear stress $\tau_{\mathrm{bn}}^{*}$ induced by the secondary flow. Olesen [1987, equation (3.18)] relates these mechanisms as

$$
\frac{q_{\mathrm{bn}}}{q_{\mathrm{bs}}}=\frac{U_{\mathrm{n}}}{U_{\mathrm{s}}}+\frac{\tau_{\mathrm{bn}}^{*}}{\tau_{\mathrm{s}}}-G \frac{\partial z_{\mathrm{b}}}{\partial n},
$$

and summarizes different models for the gravitational pull $G$. In axisymmetric curved flow (also called fully developed curved flow, defined by zero streamwise derivatives, zero cross-flow, and zero transverse sediment transport and indicated by the subscript $\infty$ ), equation (2) reduces to

$$
\left(\frac{\partial z_{\mathrm{b}}}{\partial n}\right)_{\infty}=\frac{1}{G}\left(\frac{\tau_{\mathrm{bn}}^{*}}{\tau_{\mathrm{bs}}}\right)_{\infty}
$$

indicating that the transverse bed slope is determined by the curvature-induced secondary flow. In general, the transverse bed slope scales with the inverse of the radius of curvature. Camporeale et al. [2007] identified this as the most important and most sensitive feedback mechanism between the flow and the morphology. Because the contribution of the present article concerns the hydrodynamic submodel, the reader is referred to the study of Camporeale et al. [2007] for more details on the modeling of the morphology.

\subsubsection{A Meander Hydrodynamics Submodel}

[16] The hydrodynamic model describes curvature effects on the flow field. Because the large-scale one-dimensional morphodynamic model defines the cross-sectional averaged velocity $U$, the meander hydrodynamic model has to provide the transverse structure of the flow field including the velocity excess near the outer bank $\Delta U_{\mathrm{s}}$, which drives the meander migration submodel. Moreover, it has to determine the transverse component of the bed shear stress $\tau_{\mathrm{bn}}^{*}$ induced by the secondary flow that plays a fundamental role in the meander morphology submodel.

[17] Most hydrodynamic meander models are derived from the depth-averaged conservation equations of mass and momentum, which govern the transverse structure of the flow field [Blanckaert and de Vriend, 2003]:

$$
\begin{aligned}
& \frac{1}{1+n / R} \frac{\partial U_{\mathrm{s}} h}{\partial s}+\frac{\partial U_{\mathrm{n}} h}{\partial n}+\frac{U_{\mathrm{n}} h}{(1+n / R) R}=0 \\
& \text { or } \frac{1}{1+n / R}\left(\frac{\partial U_{\mathrm{s}} h}{\partial s}+\frac{\partial}{\partial n}\left[(1+n / R) U_{\mathrm{n}} h\right]\right)=0
\end{aligned}
$$

$$
\begin{array}{r}
\frac{1}{1+n / R} \frac{\partial\left\langle v_{\mathrm{s}} v_{\mathrm{s}}\right\rangle h}{\partial s}+\frac{\partial\left\langle v_{\mathrm{s}} v_{\mathrm{n}}\right\rangle h}{\partial n}+\frac{2\left\langle v_{\mathrm{s}} v_{\mathrm{n}}\right\rangle h}{(1+n / R) R} \\
=-\frac{1}{1+n / R} g h \frac{\partial z_{\mathrm{s}}}{\partial s}-\frac{\tau_{\mathrm{bs}}}{\rho}+\mathrm{HDT}_{\mathrm{s}}
\end{array}
$$

$\mathrm{HDT}_{\mathrm{s}}$ represents turbulent diffusion terms, $z_{\mathrm{S}}$ is the water surface elevation, $\tau_{\mathrm{bs}}$ is the streamwise component of the bed shear stress, and the brackets \langle\rangle indicate depth-averaged values. Hydrostatic pressure has been assumed in the depth-averaged momentum equation (5). The highly threedimensional nature of flow in open-channel bends appears explicitly in the equations by decomposing the velocities into depth-averaged values and local spatial deviations as

$$
v_{\mathrm{i}}=\left\langle v_{\mathrm{i}}\right\rangle+v_{\mathrm{i}}^{*}=U_{\mathrm{i}}+v_{\mathrm{i}}^{*}(\mathrm{i}=\mathrm{s}, \mathrm{n}) .
$$

The depth-averaged transverse velocity $U_{\mathrm{n}}$ represents mass transport across the cross section, whereas the spatial deviations $v_{\mathrm{n}}^{*}$ represent the horizontal component of the secondary flow (also commonly called spiral flow, helical motion, or secondary circulation), which is a characteristic curvature-induced flow feature (Figure 1). This velocity decomposition allows transforming the depth-averaged momentum equation (5) into

$$
\begin{aligned}
\frac{1}{1+n / R} U_{\mathrm{s}} h \frac{\partial U_{\mathrm{s}}}{\partial s}+U_{\mathrm{n}} h \frac{\partial U_{\mathrm{s}}}{\partial n}+\frac{U_{\mathrm{s}} U_{\mathrm{n}} h}{(1+n / R) R} \\
=-\frac{1}{1+n / R} g h \frac{\partial z_{\mathrm{s}}}{\partial s}-\frac{\tau_{\mathrm{bs}}}{\rho}-\frac{1}{1+n / R} \frac{\partial\left\langle v_{\mathrm{s}}^{*} v_{\mathrm{s}}^{*}\right\rangle h}{\partial s}-\frac{\partial\left\langle v_{\mathrm{s}}^{*} v_{\mathrm{n}}^{*}\right\rangle h}{\partial n} \\
\quad-\frac{2\left\langle v_{\mathrm{s}}^{*} v_{\mathrm{n}}^{*}\right\rangle h}{(1+n / R) R}+\mathrm{HDT}_{\mathrm{s}} .
\end{aligned}
$$

The highly three-dimensional flow in meanders is an example of nonlinear mechanics, where mutual interactions exist between different variables/processes. The main nonlinear processes, represented by-product terms in equation (7), will now briefly be explained.

[18] One of the most important nonlinear feedback processes occurs between the bed morphology and the flow field. As aforementioned, the transverse bed slope in openchannel bends scales with the inverse of the radius of curvature, indicating an increasing flow depth from the inner toward the outer bank. According to Chézy's law, the depth-averaged velocity scales with the square of the flow depth, implying that higher/lower velocities will be attracted to the deeper/shallower parts of the cross section and accompanying higher/lower sediment transport, leading to a positive feedback between the depth-averaged flow field and the transverse bed slope. The interaction between the hydrodynamic and morphodynamic submodels accounts for this feedback.

[19] The second and third terms in the left-hand side of equation (7) represent nonlinear interactions between the streamwise and transverse depth-averaged velocities $\left(U_{\mathrm{s}}, U_{\mathrm{n}}\right)$ and the morphology $h$. According to the requirement of mass conservation [equation (4)], streamwise changes in bed topography cause a redistribution of flow materialized by a transverse mass flux $U_{\mathrm{n}} h$. A streamwise increase/decrease in flow depth in the outer/inner half of the cross section, e.g., will lead to outward mass transport. This phenomenon is often called topographic steering. This transverse mass flux $U_{\mathrm{n}} h$ is accompanied by a transverse advection of streamwise momentum $U_{\mathrm{s}} U_{\mathrm{n}} h$, which strengthens the transverse velocity redistribution. The inclusion of this nonlinear process in 
hydrodynamic models depends essentially on the modeling of the depth-averaged transverse velocity $U_{\mathrm{n}}$.

[20] The bracketed terms in the right-hand side of equation (7) indicate the influence of the vertical flow structure on the horizontal distribution of the flow, and especially the influence of the curvature-induced secondary flow $v_{\mathrm{n}}^{*}$. The curvature-induced secondary flow is commonly explained and modeled as resulting from the quasibalance between the outward centrifugal force and the inward pressure gradient due to the transverse tilting of the water surface. Because of inertia, the secondary flow lags behind the driving curvature. Advective transport of streamwise momentum by the secondary flow $v_{\mathrm{s}}^{*} v_{\mathrm{n}}^{*}$ causes an outward redistribution of velocities and deforms the vertical velocity profiles of $v_{\mathrm{s}}$ and $v_{\mathrm{n}}^{*}$. These modified transverse and vertical velocity distributions are known to reduce the magnitude of the secondary flow [de Vriend, 1981; Blanckaert and de Vriend, 2003; Blanckaert and Graf, 2004], indicating nonlinear feedback between the vertical profiles of the streamwise velocity and the secondary flow, as well as between the vertical and transverse structures of the flow. The major effect of the secondary flow is, however, related to the induced transverse component of the bed shear stress $\tau_{\mathrm{bn}}^{*}$, which conditions the transverse bed slope [see equations (2) and (3)] [Camporeale et al., 2007]. This additional inward component of the bed shear stress and the deformed vertical velocity profiles increase the magnitude of the bed shear stresses and the frictional energy losses. The inclusion of the influence of the vertical flow structure in the hydrodynamic meander models depends essentially on the modeling of the curvatureinduced secondary flow.

\subsection{Comparison to Other Models and Contribution of the Proposed Model}

[21] Mathematical models of meander evolution have been progressively developed and refined during the last decades [e.g., Ikeda et al., 1981; Parker et al., 1982, 1983; Howard, 1984; Blondeaux and Seminara, 1985; Parker and Andrews, 1986; Odgaard, 1989; Johannesson and Parker, 1989b; Seminara and Tubino, 1992; Liverpool and Edwards, 1995; Stølum, 1996, 1998; Imran et al., 1999; Zolezzi and Seminara, 2001; Edwards and Smith, 2002; Lancaster and Bras, 2002; Camporeale et al., 2007; Crosato, 2008; Bolla Pittaluga et al., 2009]. The hydrodynamic component of meander models is mostly based on the reduction and modeling of the depthaveraged flow equations (4), (5), and (A9). Camporeale et al. [2007] have established a general mathematical framework that allowed comparison and hierarchization of meander models. The present section intends to situate the contribution and originality of the proposed hydrodynamic model by comparing to some meander models. The comparison will highlight the adopted assumptions and approach to reduce the governing equations, the aforementioned nonlinear hydrodynamic processes, the curvature-induced energy losses, as well as the resulting scaling parameters. The comparison (summarized in Table 1) will focus on the most recent and most complete hydrodynamic meander models proposed by Odgaard [1989], Johannesson and Parker [1989b], Imran et al. [1999], Zolezzi and Seminara [2001], and Bolla Pittaluga et al. [2009].

\subsubsection{Approach to Reduce the Governing Equations}

[22] Howard [1984] and Odgaard [1989] applied an empirical approach to reduce the governing equations. On the basis of observations in natural configurations and laboratory models, they identified the processes of minor importance and neglected the corresponding terms in the governing equations and approximated the three-dimensional distribution of variables by means of profile functions with a limited degree of freedom. For example, Ikeda et al. [1981], Blondeaux and Seminara [1985], Seminara and Tubino [1992], Imran et al. [1999], Camporeale et al. [2007], Crosato [2008], and Bolla Pittaluga et al. [2009] applied a small-perturbation approach that decomposes variables into their value for a known reference state corrected by a small perturbation:

$$
\operatorname{var}=\operatorname{var}_{0}+\varepsilon \operatorname{var}_{1}+O\left(\varepsilon^{2}\right)
$$

where $\varepsilon$ is a small parameter that parameterizes curvature. Table 1 summarizes different small parameters for curvature influences that have been proposed in the literature. Applying this decomposition to a governing equation that can generally be written as

$$
F\left(\operatorname{var}^{\mathrm{a}}, \operatorname{var}^{\mathrm{b}}, \operatorname{var}^{\mathrm{c}}, \ldots\right)=0
$$

allows identifying terms of different orders of magnitude

$$
\begin{aligned}
& e^{0} F^{0}\left(\operatorname{var}_{0}^{\mathrm{a}}, \operatorname{var}_{0}^{\mathrm{b}}, \operatorname{var}_{0}^{\mathrm{c}}, \ldots\right) \\
& +\varepsilon^{1} F^{1}\left(\operatorname{var}_{0}^{\mathrm{a}}, \operatorname{var}_{0}^{\mathrm{b}}, \operatorname{var}_{0}^{\mathrm{c}}, \operatorname{var}_{1}^{\mathrm{a}}, \operatorname{var}_{1}^{\mathrm{b}}, \operatorname{var}_{1}^{\mathrm{c}}, \ldots\right) \\
& +\varepsilon^{2} F^{2}\left(\operatorname{var}_{0}^{\mathrm{a}}, \operatorname{var}_{0}^{\mathrm{b}}, \operatorname{var}_{0}^{\mathrm{c}}, \operatorname{var}_{1}^{\mathrm{a}}, \operatorname{var}_{1}^{\mathrm{b}}, \operatorname{var}_{1}^{\mathrm{c}}, \operatorname{var}_{2}^{\mathrm{a}}, \operatorname{var}_{2}^{\mathrm{b}}, \operatorname{var}_{2}^{\mathrm{c}}, \ldots\right) \\
& +O\left(\varepsilon^{3}\right) \\
& =0
\end{aligned}
$$

Most models are limited to the reference state corrected by a perturbation at order $\varepsilon$. These models are called linear models because they cannot account for nonlinear interactions between different variables. Some models have been proposed [Imran et al., 1999; Zolezzi and Seminara, 2001; Camporeale et al., 2007; Bolla Pittaluga et al., 2009] that retain higherorder contributions and can account for nonlinear interactions. The main hypothesis underlying these small-perturbation methods is that the equations can be separated in contributions of similar order of magnitude that can be solved separately subject to appropriate boundary conditions. This requires the perturbation to be small with respect to the reference state, $\varepsilon \operatorname{var}_{1} \ll \operatorname{var}_{0}$ in equation (8). Sharp natural open-channel bends are typically characterized by scaling parameters that can attain values of about $(B / 2 R) \approx 0.5, H / R \approx 0.05, C_{\mathrm{f}}^{-1 / 2} H / R \approx$ 0.5 , which invalidate the small-perturbation approach (Table 1 ). Blanckaert [2009, Figure 4], for example, shows that the perturbation of the streamwise and transverse velocity profiles can be of the same order of magnitude as the reference state in a strongly curved open-channel flow. Moreover, the order of importance of different processes in a small-perturbation approach is conditioned by the choice of the scaling parameters and the small curvature parameter. Advective momentum transport by the curvature-induced secondary flow $v_{\mathrm{n}}^{*}$ and by the topography-induced cross-flow $U_{\mathrm{n}}$, for example, is known 


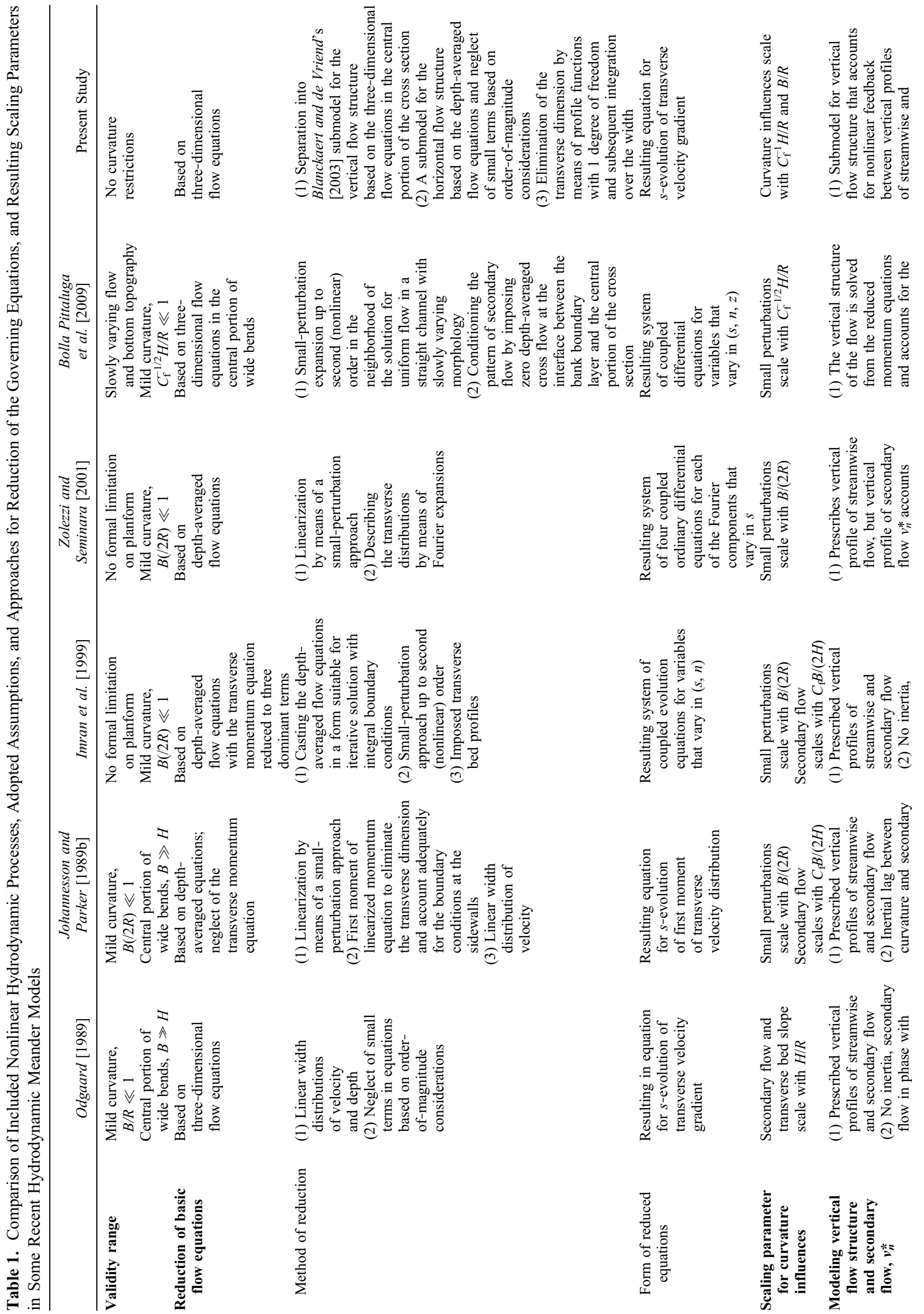




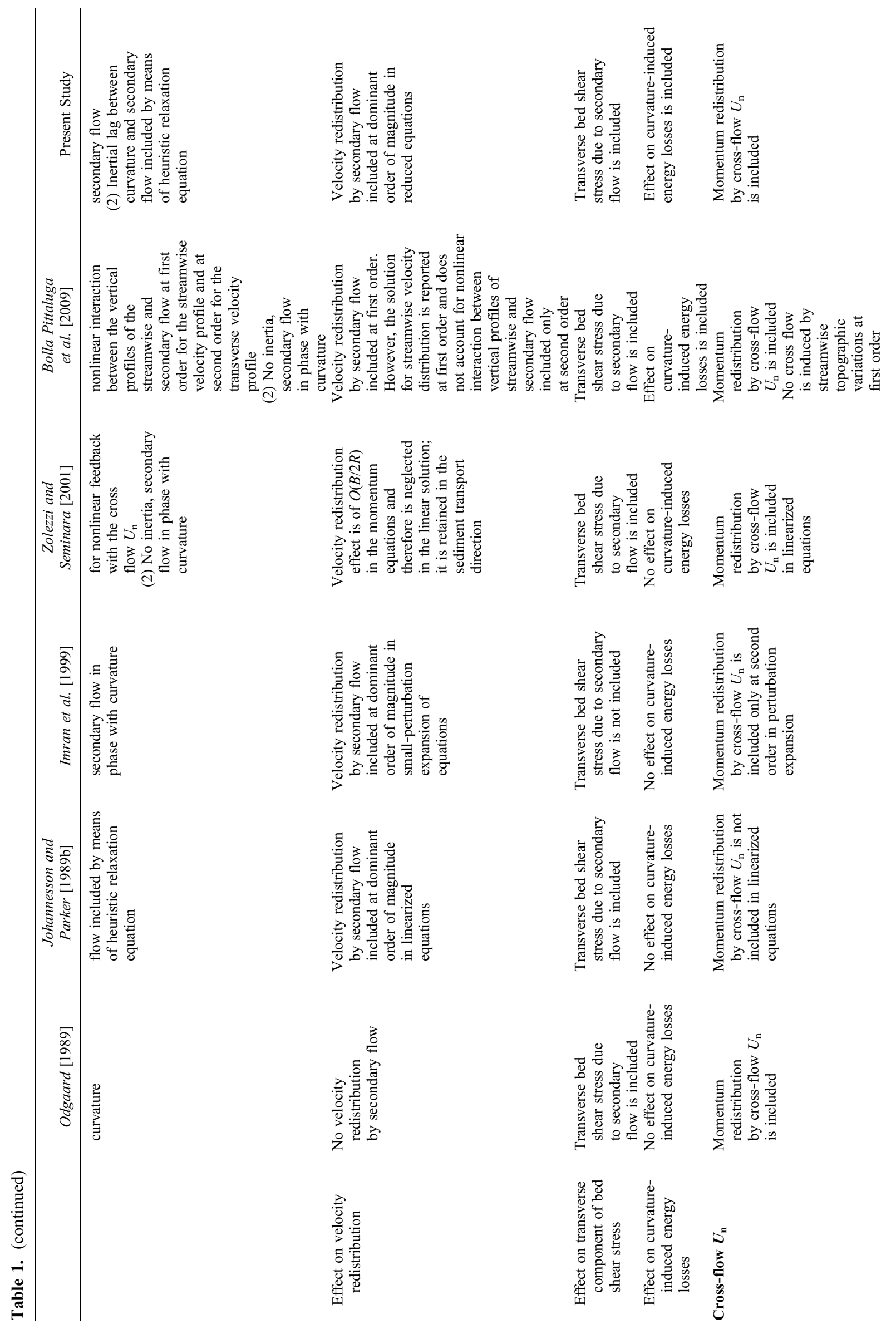




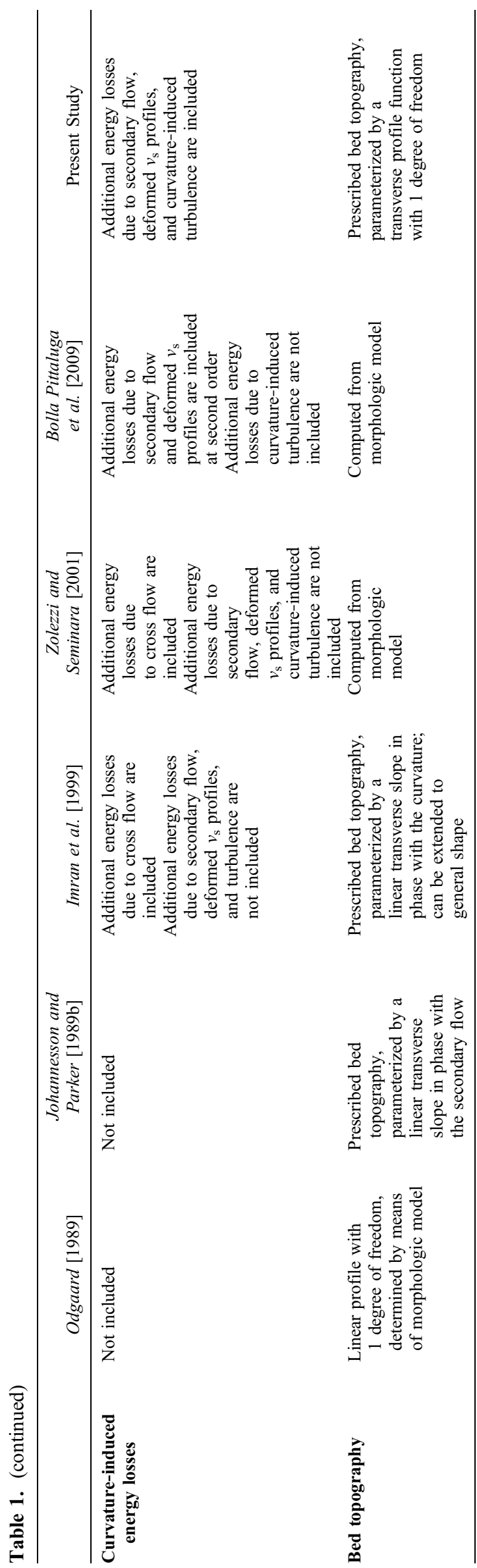

from observations in laboratory flumes and natural rivers to be of dominant order of magnitude in moderately and sharply curved open-channel bends, although these processes only occur as a lower-order contribution in some models based on small-perturbation methods (Table 1). For these reasons, the proposed model is based on an empirical approach. It approximates the three-dimensional flow patterns by means of two coupled submodels, which describe the vertical and horizontal structures of the flow field, respectively.

\subsubsection{Vertical Flow Structure and Secondary Flow}

[23] As aforementioned, Camporeale et al. [2007] have identified the transverse component of the bed shear stress induced by the secondary flow as the major and most sensitive feedback mechanism between the flow and the morphology in meanders. Therefore, the principal contribution of the proposed model is to account accurately for nonlinear hydrodynamic processes in the description of the vertical flow structure and especially of the curvature-induced secondary flow. Linear models [e.g., van Bendegom, 1947; Rozovskii, 1957; Engelund, 1974; de Vriend, 1977; Odgaard, 1989; Johannesson and Parker, 1989a] and even the nonlinear model of Imran et al. [1999] prescribe the vertical flow structure in the central portion of the cross section (Table 1): they adopt the same streamwise velocity profiles as in straight uniform flow and impose a secondary flow that grows proportionally to the ratio of the flow depth to the radius of curvature:

$$
\begin{gathered}
v_{\mathrm{s} 0}=U_{\mathrm{s}} f_{\mathrm{s} 0}\left(C_{\mathrm{f}}\right)=U_{\mathrm{s}}+U_{\mathrm{s}}\left[f_{\mathrm{s} 0}\left(C_{\mathrm{f}}\right)-1\right]=U_{\mathrm{s}}+v_{\mathrm{s} 0}^{*}\left(C_{\mathrm{f}}\right), \\
v_{\mathrm{n} 0}^{*}=U_{\mathrm{s}} \frac{h}{(1+n / R) R} f_{\mathrm{n} 0}\left(C_{\mathrm{f}}\right) .
\end{gathered}
$$

The index 0 indicates linear model functions based on the prescribed vertical profile functions $f_{\mathrm{s} 0}$ and $f_{\mathrm{n} 0}$ that depend uniquely on the friction coefficient $C_{\mathrm{f}}$. The profiles $f_{\mathrm{s}}$ and $f_{\mathrm{n}}$ fully determine the advective transport of streamwise momentum $\left\langle v_{\mathrm{s}}^{*} v_{\mathrm{n}}^{*}\right\rangle$ and the transverse component of the bed shear stress $\tau_{\mathrm{bn}}^{*}$ induced by the secondary flow in the central portion of the cross section as:

$$
\begin{gathered}
\left\langle v_{\mathrm{s}}^{*} v_{\mathrm{n}}^{*}\right\rangle=\frac{U_{\mathrm{s}}^{2} h}{(1+n / R)} \frac{\left\langle f_{\mathrm{s}} f_{\mathrm{n}}\right\rangle}{R} \text { with } \frac{\left\langle f_{\mathrm{s}} f_{\mathrm{n}}\right\rangle_{0}}{R}=f c t\left(C_{\mathrm{f}}\right) \quad \text { and } \\
\frac{\tau_{\mathrm{bn}}^{*}}{\tau_{\mathrm{bs}}}=-\frac{h}{(1+n / R)} \frac{\alpha_{\tau}}{R} \text { with } \frac{\alpha_{\tau 0}}{R}=f c t\left(C_{\mathrm{f}}\right) .
\end{gathered}
$$

The latter has been represented in a normalized form by the parameter $\alpha_{\tau}$ similar to, e.g., Engelund [1974], Kikkawa et al. [1976], de Vriend [1977], Struiksma et al. [1985]. The division of the variables $\left\langle f_{\mathrm{s}} f_{\mathrm{n}}\right\rangle$ and $\alpha_{\tau}$ by $R$ guarantees the validity of their definition in straight flow. Figure 2a shows the solutions for $\left\langle f_{\mathrm{s}} f_{\mathrm{n}}\right\rangle_{0}$ and $\alpha_{\tau 0}$ according to the linear model of de Vriend [1977].

[24] As mentioned before, nonlinear hydrodynamic interactions are known to deform the streamwise velocity profile and to limit the growth of the secondary flow with increasing curvature. Blanckaert [2009] shows that the secondary flow 

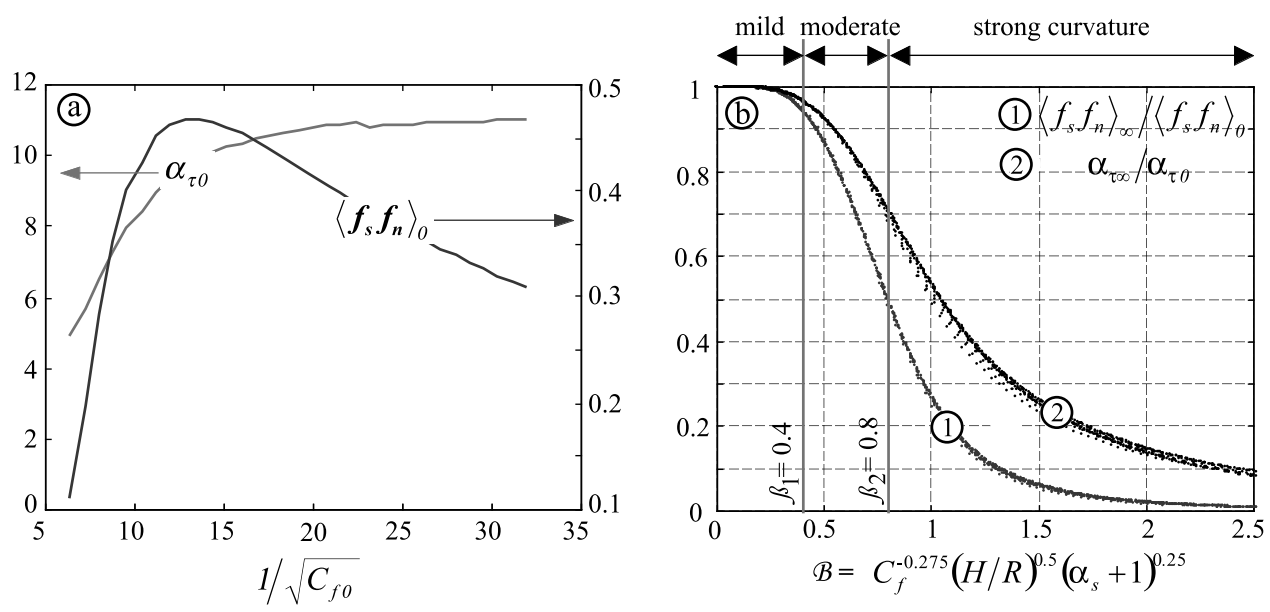

Figure 2. Advective momentum transport and transverse bed shear stress induced by the secondary flow. (a) According to the linear model of de Vriend [1977]. (b) Correction coefficient accounting for nonlinear hydrodynamic interactions according to Blanckaert and de Vriend [2003]. Modified from Figures 4 and 10 of Blanckaert and de Vriend [2003], respectively.

does not even grow any more when the curvature is increased in very sharp bends, and he called this process the saturation of the secondary flow. Hence, models that impose the vertical flow structure overestimate the secondary flow, as well as its induced advective momentum transport [equation (13)] and transverse bed shear stress [equation (14)]. The importance of the nonlinear hydrodynamics on the secondary flow effects was recognized by Zolezzi and Seminara [2001] and Bolla Pittaluga et al. [2009]. Zolezzi and Seminara [2001] account for the interaction between the curvature-induced secondary flow $v_{\mathrm{n}}^{*}$ and the topography-induced cross-flow $U_{\mathrm{n}}$ but still neglect the dominant interaction with the streamwise velocity. Bolla Pittaluga et al. [2009] take it into account by reducing the fully three-dimensional flow equations based on a smallperturbation approach. However, the deformation of the vertical profiles of streamwise velocity and secondary flow, as well as its effect on the velocity redistribution and the transverse bed shear stress, is only accounted for from the second order of approximation $\left[\varepsilon^{2}\right.$ in equation (10)]. Moreover, their patterns of secondary flow seem to be considerably conditioned by the imposition of zero depth-averaged cross-flow at the interface between the bank boundary layer and the central portion of the cross section.

[25] As mentioned before, one of the principal contributions of the proposed model is to account accurately for nonlinear hydrodynamic processes in the description of the vertical flow structure by means of Blanckaert and de Vriend's [2003] model without curvature restrictions. Instead of using predefined velocity profiles, or profiles with 1 degree of freedom, this model determines the entire vertical profiles of the streamwise velocity and the secondary flow from the threedimensional flow equations in the central portion of the cross section. The solutions of the vertical profiles can only be obtained numerically and depend on the friction coefficient $C_{\mathrm{f}}$, the ratio of local flow depth to radius of curvature $h /[(1+n / R) R]$, and the coefficient $\alpha_{\mathrm{s}}$, which is defined as

$$
\alpha_{\mathrm{s}}=\frac{(1+n / R) R}{U_{\mathrm{s}}} \frac{\partial U_{\mathrm{s}}}{\partial n},
$$

and parameterizes the transverse velocity distribution, thus providing the feedback between the vertical and the transverse structures of the flow. When plotted against the combined parameter, called the bend parameter,

$$
\mathscr{B}=\left(C_{\mathrm{f}}\right)^{-0.275}\{h /[(1+n / R) R]\}^{0.5}\left(\alpha_{\mathrm{s}}+1\right)^{0.25},
$$

the solutions for the advective momentum transport and the transverse bed shear stress almost collapse on a single curve (Figure $2 \mathrm{~b}$ ) when normalized by the linear model solutions (Figure 2a):

$$
\begin{aligned}
\left\langle v_{\mathrm{s}}^{*} v_{\mathrm{n}}^{*}\right\rangle & =\frac{U_{\mathrm{s}}^{2} h}{(1+n / R)} \frac{\left\langle f_{\mathrm{s}} f_{\mathrm{n}}\right\rangle}{R} \text { with } \frac{\left\langle f_{\mathrm{s}} f_{\mathrm{n}}\right\rangle_{\infty}}{R} / \frac{\left\langle f_{\mathrm{s}} f_{\mathrm{n}}\right\rangle_{0}}{R} \\
& =f c t(B) \text { and } \\
\frac{\tau_{\mathrm{bn}}^{*}}{\tau_{\mathrm{bs}}}= & -\frac{h}{(1+n / R)} \frac{\alpha_{\tau}}{R} \text { with } \frac{\alpha_{\tau \infty}}{R} / \frac{\alpha_{\tau 0}}{R}=f c t(B) .
\end{aligned}
$$

This implies that no numerical solution of the entire vertical velocity profiles is required but that the nonlinear model solutions are simply obtained by computing the linear model solutions (index 0, Figure 2a) and subsequently applying a nonlinear correction factor according to the curves shown in Figure 2b. Hence, this nonlinear model is computationally hardly more expensive than linear models. Thanks to this nonlinear correction factor, the secondary flow and its induced advective momentum transport and transverse bed shear stress do not grow proportionally to the curvature ratio $h /[(1+$ $n / R) R]$ anymore. The model even captures the saturation of the secondary flow effects in very sharp open-channel bends [Blanckaert, 2009; K. Blanckaert, Hydrodynamic processes in sharp meander bends and their morphological 
implications, submitted to Journal of Geophysical Research, 2010].

[26] The index $\infty$ in equations (17) and (18) indicates that Blanckaert and de Vriend's [2003] model neglects inertial effects, which cause the secondary flow and its effects to lag behind the driving curvature. This phase lag has been neglected in most meander models (Table 1) and has negligible effect on the meander processes according to Camporeale et al. [2007]. Because it might have nonnegligible effect in sharply curved open-channel bends with pronounced curvature variations, the proposed model accounts for this inertial phase lag by means of the same heuristic relaxation equation used by Johannesson and Parker [1989a]:

$$
\begin{gathered}
\lambda \frac{\partial Y}{\partial s}+Y=Y_{\infty} \text { and } \\
\lambda=\frac{H}{\delta C_{\mathrm{f}}} \text { with } \delta=\frac{\left(13 \sqrt{C_{\mathrm{f}}}\right)^{-2}\left[\left(13 \sqrt{C_{\mathrm{f}}}\right)^{-1}-\frac{1}{12}\right]}{\frac{\left(13 \sqrt{C_{\mathrm{f}}}\right)^{2}}{12}-\frac{\left(13 \sqrt{C_{\mathrm{f}}}\right)^{-1}}{40}+\frac{1}{945}} .
\end{gathered}
$$

where $Y=\left\langle f_{\mathrm{s}} f_{\mathrm{n}}\right\rangle / R$ or $\alpha_{\tau} / R$.

\subsubsection{Curvature-Induced Energy Losses}

[27] Curvature-induced additional energy losses have been estimated and parameterized by Rozovskii [1957] and Chang [1983] but are not or only partially accounted for in most existing meander models (Table 1). On the basis of the hypothesis that the dimensionless Chézy coefficient $C_{\mathrm{f}}$ is constant in the cross section, the energy gradient $E_{\mathrm{s}}$ can be approximated as

$$
\begin{aligned}
& E_{\mathrm{s}}=\frac{\left\langle\left\langle\left|\vec{\tau}_{b}\right|\right\rangle\right\rangle}{\rho g R_{h}}=\frac{C_{\mathrm{f}}\left\langle\left\langle|\vec{U}|^{2}\right\rangle\right\rangle}{g R_{h}} \text { with } \\
& C_{\mathrm{f}}=\psi C_{\mathrm{f} 0}=\left(\psi_{\text {secondary flow }} \psi_{\alpha_{\mathrm{s}}} \psi_{\text {turbulence }}\right) C_{\mathrm{f} 0},
\end{aligned}
$$

where $\langle\langle\rangle\rangle$ represents cross-sectional averaged values, $\left|\vec{\tau}_{b}\right|$ represents the magnitude of the boundary shear stress vector, and the index 0 represents a value in straight flow. Curvature induces additional energy losses due to (1) the additional transverse component of the bed shear stress induced by the secondary flow; (2) the increased nearbed gradient of the velocity vector due to the deformed vertical velocity profiles; (3) the nonuniform distribution of the depth-averaged velocity, which implies $\left.\left\langle\left\langle\mid \vec{U}^{2} I\right\rangle\right\rangle\right\rangle$ $U^{2}$; and (4) the increased production rate of turbulence, which is, by definition, an increased loss of mean flow kinetic energy. Blanckaert and de Vriend's [2003, Figure 10] nonlinear model for curved flow quantifies the first two mechanisms by means of a correction factor $\psi_{\text {secondaryflow }}$ to the friction coefficient, which can be expressed as a function of $B C_{\mathrm{f}}^{-0.15}$ :

$$
\psi_{\text {secondary flow }}=f c t\left(B C_{\mathrm{f}}^{0.15}\right)
$$

The relevance of the third mechanism can be estimated based on a linearization of the velocity distribution according to equation (15): $U_{\text {linear }}(s, n)=U(s)\left(1+\alpha_{\mathrm{s}}(s) n / R\right)$. Averaging over the cross section (the average is in fact taken over a bend sector in order to take into account that the arc length is longer/shorter in the outer/inner part of the cross section than on the centerline), gives

$$
\psi_{\alpha_{\mathrm{s}}}=\frac{\left\langle\left\langle|\vec{U}|^{2}\right\rangle\right\rangle}{U^{2}}=1+\frac{1}{12}\left(\frac{B^{2} \alpha_{\mathrm{s}}^{2}}{R^{2}}+2 \frac{B}{R} \frac{B \alpha_{\mathrm{s}}}{R}\right) .
$$

Finally, Blanckaert [2009] developed a simple model for the curvature-induced increase in turbulence production, which is represented by means of a correction factor $\psi_{\text {turbulence. }}$.

\subsubsection{Reduction of the Flow Equations}

[28] Unlike most existing meander models, which directly model the depth-averaged flow equations [see equations (4), (5), and (A9)], the proposed nonlinear model is based on the development and modeling of a transport equation for the normalized transverse velocity gradient $\alpha_{\mathrm{s}}$ [equation (15)], which, using Blanckaert and de Vriend's [2003] nonlinear model for the vertical flow structure, has been identified as the principal control parameter for the nonlinear interaction between the vertical and the transverse structures of the flow [see equations (16), (17), and (18)]. Moreover, substitution of the definition of $\alpha_{\mathrm{s}}$ [equation (15)] transforms the streamwise momentum equation (7) into

$$
\begin{aligned}
\frac{1}{1+n / R} U_{\mathrm{s}} & \frac{\partial U_{\mathrm{s}}}{\partial s}+U_{\mathrm{s}} U_{\mathrm{n}} \frac{\alpha_{\mathrm{s}}+1}{(1+n / R) R} \\
\approx & -\frac{1}{1+n / R} g \frac{\partial z_{\mathrm{s}}}{\partial s}-\frac{\tau_{\mathrm{bs}}}{\rho h}-\frac{\partial\left\langle v_{\mathrm{s}}^{*} v_{\mathrm{n}}^{*}\right\rangle}{\partial n} \\
& -\left\langle v_{\mathrm{s}}^{*} v_{\mathrm{n}}^{*}\right\rangle\left[\frac{1}{h} \frac{\partial h}{\partial n}+\frac{2}{(1+n / R) R}\right]
\end{aligned}
$$

showing that $\alpha_{\mathrm{s}}$ also parameterizes the nonlinearities between the depth-averaged velocities.

[29] The main goal of the proposed model is not to describe the flow-bed interactions that occur at a microscale or mesoscale (e.g., ripples and dunes) but rather to focus on the global velocity pattern and the macroscale bed forms that have typical length scales of the order of the channel width. Therefore, the proposed meander model reduces the governing equations by adopting simplified width distributions of the bed level $z_{\mathrm{b}}$ and the depth-averaged streamwise velocity $U_{\mathrm{s}}$ in the form of profile functions with 1 degree of freedom, parameterized by the so-called scour factor $A$ and the normalized transverse velocity gradient $\alpha_{\mathrm{s}}$ [equation (15)], respectively:

$$
\begin{gathered}
\frac{\partial z_{\mathrm{b}}}{\partial n}=-A \frac{h}{(1+n / R) R} \approx-A \frac{H}{R} \text { and } \\
\frac{\partial U_{\mathrm{s}}}{\partial n} \stackrel{\text { Eq.(15) }}{=} \alpha_{\mathrm{s}} \frac{U_{\mathrm{s}}}{(1+n / R) R} \approx \alpha_{\mathrm{s}} \frac{U_{\mathrm{s}}}{R} .
\end{gathered}
$$


The first equality in equations (25) and (26) defines formally the coefficients $A$ and $\alpha_{\mathrm{s}}$, whereas the second equality represents linearized approximations. The adoption of such simplified transverse distributions does not preclude the proposed model to account for the nonlinear processes described before because these processes typically occur at length scales of the order of the channel width.

[30] The approach of adopting transverse profile functions with 1 degree of freedom for the bed topography has been commonly applied in the past. Only the recent nonlinear model by Bolla Pittaluga et al. [2009] attempts a more detailed transverse description of the bed topography and the velocity, based on the hypotheses that are only valid in the central portion of wide bends and the imposition of boundary conditions at the interface between the bank boundary layer and the central portion of the cross section. van Bendegom [1947], Engelund [1974], Kikkawa et al. [1976], and Odgaard [1981a] imposed exponential profile functions for the transverse bed profile [corresponding to the first equality in equation (24)], whereas Ikeda et al. [1981], Odgaard [1989], Johannesson and Parker [1989b], and Imran et al. [1999] imposed linear profile functions [corresponding to the second equality in equation (24)]. Struiksma et al. [1985], Blondeaux and Seminara [1985], and Seminara and Tubino [1992] imposed another type of profile functions of the following form:

$$
\frac{\partial^{2}}{\partial n^{2}}\left[(1+n / R) R \frac{\partial z_{\mathrm{b}}}{\partial n}\right]=A(1+n / R) R \frac{\partial z_{\mathrm{b}}}{\partial n}
$$

which includes sinusoidal functions. Zolezzi and Seminara [2001] extended this approach by expanding the transverse bed profile in a Fourier series of sinusoidal components.

[31] The distribution of the velocity in sharp open-channel bends is mostly characterized by a core of maximum velocity that migrates from one bank to another [Zeng et al., 2008; Blanckaert, 2010; Blanckaert et al., 2010]. Although it is more complex and shows more variation than that of the bed topography, it is also commonly described in meander models by means of profile functions with 1 degree of freedom. Engelund [1974] imposed exponential profile functions for the width distribution of the velocity distribution in his first-order approximation and linear ones in his secondorder approximation. Kikkawa et al. [1976] imposed exponential profiles with $\alpha_{\mathrm{s}}=1$ corresponding to a forced vortex distribution. Johannesson and Parker [1989b] and Odgaard [1989] applied linear transverse distributions of the velocity, which the latter justified by observations in mildly and moderately curved open-channel bends in the laboratory and the field. Parker and Johannesson's [1989] smallperturbation approach led to a linear profile function at first order corrected by a sinusoidal function at second order of approximation, whereas the nonlinear model of Imran et al. [1999] resulted in a parabolic correction at the nonlinear level to a linear transverse velocity distribution obtained at the linear level. Similar to the treatment of the bed level, Zolezzi and Seminara [2001] extended this approach by expanding the transverse velocity profile in a Fourier series of sinusoidal components.
[32] An analysis of the sharply curved laboratory experiments of Blanckaert and Graf [2001], Zeng et al. [2008], and Blanckaert [2010] has indicated that the linear, exponential, and sinusoidal profile functions describe the bed level and the velocity distribution with similar accuracy (see further in Figure 4); therefore, the proposed model adopts the simplest linear transverse distributions of the bed level and the velocity profile.

\section{Model Development}

[33] An evolution equation will be derived for $\alpha_{s} /[(1+n /$ $R) R]=\left(\partial U_{\mathrm{s}} / \partial n\right) / U_{\mathrm{s}}$ [equation (15)]. The denominator in the left-hand side guarantees that that the definition and the evolution equation remain valid for the asymptotic case of straight flow.

$$
\frac{\partial}{\partial s}\left[\frac{\alpha_{\mathrm{s}}}{(1+n / R) R}\right]=\frac{1}{U_{\mathrm{s}}} \frac{\partial^{2} U_{\mathrm{s}}}{\partial s \partial n}-\frac{\alpha_{\mathrm{s}}}{(1+n / R) R} \frac{1}{U_{\mathrm{s}}} \frac{\partial U_{\mathrm{s}}}{\partial s} .
$$

Because the depth-averaged continuity and momentum equations, written in a curvilinear reference system in equations (4) and (7), govern the depth-averaged flow field, they enable developing both terms in the right-hand side of equation (28). When neglecting small terms in the depthaveraged momentum equations and expressing terms related to the vertical structure of the flow field according to Blanckaert and de Vriend's [2003] model, equation (28) can be elaborated in the following form (details of the derivation are given in Appendix A):

$$
\begin{aligned}
& \frac{\partial}{\partial s}\left[\frac{\alpha_{\mathrm{s}}}{(1+n / R) R}\right]= \\
& -\frac{1}{U_{\mathrm{s}}} \frac{\partial}{\partial n}\left(U_{\mathrm{n}} \frac{\alpha_{\mathrm{s}}+1}{R}\right)+\frac{\alpha_{\mathrm{s}}}{(1+n / R) R} \frac{\alpha_{\mathrm{s}}+1}{R} \frac{U_{\mathrm{n}}}{U_{\mathrm{s}}} \\
& -\frac{g}{U_{\mathrm{s}}^{2}} \frac{\partial^{2} z_{\mathrm{s}}}{\partial s \partial n}+\frac{2 \alpha_{\mathrm{s}}}{(1+n / R) R} \frac{g}{U_{\mathrm{s}}^{2}} \frac{\partial z_{\mathrm{s}}}{\partial s} \\
& +(1+n / R) \frac{\psi C_{\mathrm{f}}}{h}\left[\frac{1}{h} \frac{\partial h}{\partial n}-\frac{1}{(1+n / R) R}\right] \\
& +\frac{U^{2} H\left\langle f_{\mathrm{s}} f_{\mathrm{n}}\right\rangle}{U_{\mathrm{s}}^{2} R} g_{\mathrm{sn}}\left\{\frac{2 \alpha_{\mathrm{s}}-1}{R} g_{\mathrm{sn}}\left(\frac{1}{g_{\mathrm{sn}}} \frac{\partial g_{\mathrm{sn}}}{\partial n}+\frac{1}{h} \frac{\partial h}{\partial n}+\frac{2}{(1+n / R) R}\right)\right. \\
& \left.-(1+n / R) \frac{1}{g_{\mathrm{sn}}} \frac{\partial}{\partial n}\left[g_{\mathrm{sn}}\left(\frac{1}{g_{\mathrm{sn}}} \frac{\partial g_{\mathrm{sn}}}{\partial n}+\frac{1}{h} \frac{\partial h}{\partial n}+\frac{2}{(1+n / R) R}\right)\right]\right\}
\end{aligned}
$$

Equation (29) clearly reveals the physical processes that redistribute the velocity in a river. The first line after the equals symbol represents the effect of the cross-flow $U_{n}$; the second line represents the effect of streamwise and transverse gradients in the water surface topography; the third line represents the effects of bed friction and gradients in the bed topography; and the fourth and fifth lines represent the effect of advective velocity redistribution by the secondary flow. Equation (29) will now be reduced by means of: 
[34] 1. Modeling the water surface topography terms in the second line of equation (29), as detailed in Appendix A.

[35] 2. Eliminating the transverse dimension by assuming the simplified transverse profiles of the bed topography $z_{\mathrm{b}}$ [equation (25)] and the depth-averaged streamwise velocity $U_{\mathrm{s}}$ [equation (26)]. On the basis of equations (25) and (A10), a similar simplified transverse profile of the flow depth can be derived as follows:

$$
\begin{aligned}
\frac{1}{h} \frac{\partial h}{\partial n} & =\frac{1}{h}\left(\frac{\partial z_{\mathrm{s}}}{\partial n}-\frac{\partial z_{\mathrm{b}}}{\partial n}\right)=\frac{U_{\mathrm{s}}^{2} / g h}{\left(1+n / R_{\mathrm{s}}\right) R_{\mathrm{s}}}+\frac{A}{(1+n / R) R} \\
& \approx \frac{S_{\mathrm{n}} F r^{2}}{\left(1+n / R_{\mathrm{s}}\right) R_{\mathrm{s}}}+\frac{A}{(1+n / R) R},
\end{aligned}
$$

where $S_{\mathrm{n}}$ is a correction factor of $O(1)$, the Froude number $\mathrm{Fr}$ is based on cross-sectional averaged values and defined as $U /(g H)^{1 / 2}$, and $R_{\mathrm{S}}$ is the streamline curvature at the centerline. According to Odgaard [1981a] and Ikeda et al. [1981], $A$ is typically in the range 2.5 to 6 for natural streams, indicating that the effect of the transverse tilting of the water surface [first contribution in equation (30)] is only relevant for configurations with immobile horizontal bed. This observation allows approximation $R_{\mathrm{s}}$ by $R$, which allows converting equation (30) similar to equations (25) and (26) into:

$$
\frac{\partial h}{\partial n}=\left(S_{\mathrm{n}} F r^{2}+A\right) \frac{h}{(1+n / R) R} \approx\left(S_{\mathrm{n}} F r^{2}+A\right) \frac{H}{R} .
$$

[36] 3. Modeling the cross-flow $U_{\mathrm{n}}$ and the cross flow terms in the first line of equation (29), based on the depthaveraged continuity equation [equation (4)] and the simplified distributions of $U_{\mathrm{s}}$ and $h$, equations (26) and (30), respectively, as detailed in Appendix A. The cross flow is 0 at the impermeable banks and reaches at the centerline a value of [equation (A17)]

$$
U_{\mathrm{n}}(n=0)=\frac{U B^{2}}{8} \frac{\partial}{\partial s}\left(\frac{\alpha_{\mathrm{s}}+S_{\mathrm{n}} F r^{2}+A}{R}\right)
$$

As expected, an outward cross flow is caused by an increase/decrease of the streamwise velocity $\left(\alpha_{\mathrm{s}}\right)$ and/or the flow depth $\left(S_{\mathrm{n}} F r^{2}+A\right)$ in the outer/inner half of the cross section.

[37] Substitution of equations (26), (30), (A10), (A14), and (A17) in equation (29) gives the modeled transport equation for $\alpha_{\mathrm{s}} /[(1+n / R) R]$.

$$
\begin{aligned}
& \frac{\partial}{\partial s}\left[\frac{\alpha_{\mathrm{s}}}{(1+n / R) R}\right]=\frac{U}{2 U_{\mathrm{s}}} \frac{\alpha_{\mathrm{s}}+1}{R} \frac{\partial}{\partial s}\left(\frac{\alpha_{\mathrm{s}}+S_{\mathrm{n}} F r^{2}+A}{R}\right) \\
& \cdot\left\{\left[\frac{1+S_{\mathrm{n}} F r^{2}+A}{R}\left(-3 n^{2}+\frac{B^{2}}{4}\right)+2 n\right]\right. \\
& \left.-\frac{\alpha_{\mathrm{s}}}{(1+n / R) R}\left(n^{2}-\frac{B^{2}}{4}\right)\left(1-\frac{1+S_{\mathrm{n}} F r^{2}+A}{R} n\right)\right\} \\
& -\frac{\partial}{\partial s}\left(\frac{1}{R_{\mathrm{s}}}\right)\left\{\frac{1}{\left(1+n / R_{\mathrm{s}}\right)^{2}}\left[1-n \frac{2 \alpha_{\mathrm{s}}}{(1+n / R) R}\right]\right\} \\
& -\frac{2 \alpha_{\mathrm{s}}}{(1+n / R) R} \frac{U^{2}}{U_{\mathrm{s}}^{2}} \frac{\psi C_{\mathrm{f}}}{H} \\
& +(1+n / R) \frac{\psi C_{\mathrm{f}}}{h}\left[\frac{S_{\mathrm{n}} F r^{2}+A-1}{(1+n / R) R}\right] \\
& +\frac{U^{2} H\left\langle f_{\mathrm{s}} f_{\mathrm{n}}\right\rangle}{U_{\mathrm{s}}^{2} R} g_{\mathrm{sn}}\left\{\frac{2 \alpha_{\mathrm{s}}-1}{R}\left[\frac{1}{g_{\mathrm{sn}}} \frac{\partial g_{\mathrm{sn}}}{\partial n}+\frac{S_{\mathrm{n}} F r^{2}+A+2}{(1+n / R) R}\right]\right. \\
& \left.-(1+n / R) \frac{1}{g_{\mathrm{sn}}} \frac{\partial}{\partial n}\left[g_{\mathrm{sn}}\left(\frac{1}{g_{\mathrm{sn}}} \frac{\partial g_{\mathrm{sn}}}{\partial n}+\frac{S_{\mathrm{n}} F r^{2}+A+2}{(1+n / R) R}\right)\right]\right\} .
\end{aligned}
$$

[38] The transport equation for $\alpha_{\mathrm{s}} /[(1+n / R) R]$ is further reduced by averaging equation (33) over the width of the river, resulting in the following transport equation for $\alpha_{\mathrm{s}} / R$ :

$$
\begin{aligned}
& \frac{\partial}{\partial s}\left(\frac{\alpha_{\mathrm{s}}}{R}\right)=\frac{1}{12} \frac{\alpha_{\mathrm{s}}+1}{R} \frac{B^{2}}{R} \frac{\partial}{\partial s}\left(\frac{\alpha_{\mathrm{s}}+S_{\mathrm{n}} F r^{2}+A}{R}\right) \\
& -\left(1+\frac{B^{2}}{6} \frac{2 \alpha_{\mathrm{s}}-1}{R^{2}}\right) \frac{\partial}{\partial s}\left(\frac{1}{R}\right)-\frac{2 \alpha_{\mathrm{s}}}{R} \frac{\psi C_{\mathrm{f}}}{H} \\
& +\frac{\psi C_{\mathrm{f}}}{H} \frac{S_{\mathrm{n}} F r^{2}+A-1}{R} \\
& +\frac{2 \chi}{3} \frac{H\left\langle f_{\mathrm{s}} f_{\mathrm{n}}\right\rangle}{R}\left[\left(\frac{2 \alpha_{\mathrm{s}}-1}{R}\right)^{2}+\frac{1}{R} \frac{S_{\mathrm{n}} F r^{2}+A+2}{R}+\frac{12}{B^{2}}\right] .
\end{aligned}
$$

Details of this operation and the hypothesis involved are summarized in Appendix A. The four lines still represent the same four physical processes described in equation (29). It is convenient to write this equation in the form of a relaxation equation with adaptation length $\lambda_{\alpha s / R}$ and driving mechanism $F_{\alpha \mathrm{s} / R}$ :

$$
\begin{gathered}
\lambda_{\alpha \mathrm{s} / R} \frac{\partial}{\partial \mathrm{s}}\left(\frac{\alpha_{\mathrm{s}}}{R}\right)+(1+\varepsilon) \frac{\alpha_{\mathrm{s}}}{R}=F_{\alpha \mathrm{s} / R}, \\
\lambda_{\alpha \mathrm{s} / R}=\frac{1}{2} \frac{H}{\psi C_{\mathrm{f}}}\left(1-\frac{1}{12} \frac{\alpha_{\mathrm{s}}+1}{R} \frac{B^{2}}{R}\right), \text { and }
\end{gathered}
$$

$$
\begin{aligned}
& \mathrm{F}_{\alpha \mathrm{s} / R}=\frac{1}{2} \frac{S_{\mathrm{n}} F r^{2}+A-1}{R}-\frac{1}{2} \frac{H}{\psi C_{\mathrm{f}}} \frac{\partial}{\partial s}\left(\frac{1}{R_{\mathrm{s}}}\right)\left(1-\frac{B^{2}}{6 R^{2}}\right)+\frac{4 \chi}{\psi C_{\mathrm{f}}} \frac{H^{2}}{B^{2}} \frac{\left\langle f_{\mathrm{s}} f_{\mathrm{n}}\right\rangle}{R}\left[1+\frac{1}{12} \frac{\left(S_{\mathrm{n}} F r^{2}+A+3\right) B^{2}}{R^{2}}\right]+\frac{1}{24} \frac{\mathrm{H}}{\psi C_{\mathrm{f}}} \frac{B^{2}}{R^{2}} \frac{\partial}{\partial s}\left(\frac{S_{\mathrm{n}} F r^{2}+A}{R}\right) \\
& =\mathrm{I}+\mathrm{I}+\mathrm{II}+\mathrm{III} \quad+\quad \text { IV }
\end{aligned}
$$


The term $\varepsilon$ in equation (35) is defined as:

$$
\begin{aligned}
\varepsilon & =-\frac{1}{24} \frac{H}{\psi C_{\mathrm{f}}}\left[\frac{B^{2}}{R} \frac{\partial}{\partial s}\left(\frac{S_{\mathrm{n}} F r^{2}+A}{R}-\frac{4}{R_{\mathrm{s}}}\right)+\frac{32 \chi H\left\langle f_{\mathrm{s}} f_{\mathrm{n}}\right\rangle}{R} \frac{\alpha_{\mathrm{s}}-1}{\mathrm{R}}\right] \\
& =-\frac{1}{24}\left(\frac{H}{C_{\mathrm{f}} B}\right)\left(\frac{B}{R}\right)^{2}\left[O\left(\frac{B}{\lambda_{\mathrm{A}}}\right)+O\left(10 \alpha_{\mathrm{s}} \frac{H}{B}\right)\right] \\
& =-\frac{1}{24}\left(\frac{H}{C_{\mathrm{f}} B}\right)\left(\frac{B}{R}\right)^{2} O(1) .
\end{aligned}
$$

The characteristic length scale for variations of the transverse bed slope and the radius of curvature, $\lambda_{A}$, is of the order of the radius of curvature $R$. The parameter $C_{\mathrm{f}}^{-1} H / B$ reaches maximum values at bankfull discharge, which are typically about 5 to 10 (K. Blanckaert, submitted manuscript, 2010). This order-of-magnitude analysis indicates that the term $\varepsilon$ is negligible in mildly and moderately curved openchannel bends but might be relevant in very sharply curved bends. This term was found not to significantly influence the proposed model's results in sharp bends but to deteriorate its stability (not shown). Therefore, the term $\varepsilon$ has been neglected further on. The first term (I) in equation (37) represents the effect of the transverse slopes of the bed and the water surface, the second term (II) represents local flow accelerations/decelerations due to the adaptation of the transverse water surface slope to changes in curvature, the third term (III) represents velocity redistribution by the secondary flow, and the fourth term (IV) represents velocity redistribution by the cross-flow $U_{\mathrm{n}}$ resulting from streamwise variations in the transverse slopes of the bed and the water surface.

[39] Solution of this equation requires an expression for the streamline curvature at the centerline $R_{\mathrm{s}}$, which is obtained by applying the definition of curvature as

$$
\begin{aligned}
\frac{\sqrt{U_{\mathrm{s}}^{2}+U_{\mathrm{n}}^{2}}}{(1+n / R) R_{\mathrm{s}}}= & U_{\mathrm{n}}\left[\frac{1}{1+n / R} U_{\mathrm{s}} \frac{\partial U_{\mathrm{s}}}{\partial s}+U_{\mathrm{n}} \frac{\partial U_{\mathrm{s}}}{\partial n}+\frac{U_{\mathrm{s}} U_{\mathrm{n}}}{(1+n / R) R}\right] \\
& -U_{\mathrm{s}}\left[\frac{1}{1+n / R} U_{\mathrm{s}} \frac{\partial U_{\mathrm{n}}}{\partial s}+U_{\mathrm{n}} \frac{\partial U_{\mathrm{n}}}{\partial n}-\frac{U_{\mathrm{s}}^{2}}{(1+n / R) R}\right] .
\end{aligned}
$$

The first and second terms between brackets represent streamwise and transverse accelerations, respectively [see equations (7) and (A9)]. Applying this equation at the centerline and assuming that the terms $U_{\mathrm{n}}\left(\partial U_{\mathrm{n}} / \partial n\right)$ and $\partial U_{\mathrm{s}} / \partial s$ are negligible at the centerline, that $\sqrt{U_{\mathrm{s}}^{2}+U_{\mathrm{n}}^{2}} \approx U_{\mathrm{s}}^{2} \approx U^{2}$, and substituting the definition of $\alpha_{s} /[(1+n / R) R]$ results in the following expression for the difference between streamline curvature and geometric river curvature at the centerline:

$$
\begin{aligned}
\frac{1}{R_{\mathrm{s}}}-\frac{1}{R}= & \frac{\alpha_{\mathrm{s}}+1}{R}\left[\frac{B^{2}}{8} \frac{\partial}{\partial s}\left(\frac{\alpha_{\mathrm{s}}+S_{\mathrm{n}} F r^{2}+A}{R}\right)\right]^{2} \\
& -\frac{B^{2}}{8} \frac{\partial^{2}}{\partial s^{2}}\left(\frac{\alpha_{\mathrm{s}}+S_{\mathrm{n}} F r^{2}+A}{R}\right) \\
\frac{\frac{1}{R_{\mathrm{s}}}-\frac{1}{R}}{\frac{1}{R}}= & O\left(\alpha_{\mathrm{s}}\right)\left[\frac{B^{2}}{8 R \lambda_{\mathrm{A}}} O\left(\alpha_{\mathrm{s}}+A\right)\right]^{2}-\frac{B^{2}}{8 \lambda_{A}^{2}} O\left(\alpha_{\mathrm{s}}+A\right) \\
= & \left(\frac{B}{R}\right)^{2}\left[\left(\frac{B}{R}\right)^{2} O\left(\frac{1}{10}\right)-O\left(\frac{1}{10}\right)\right]
\end{aligned}
$$

This order-of-magnitude analysis indicates that the difference between the geometric curvature and the streamline curvature at the centerline is not relevant. This difference has also been neglected in meander models of Odgaard [1989], Johannesson and Parker [1989b], Imran et al. [1999], Zolezzi and Seminara [2001], and Bolla Pittaluga et al. [2009]. This difference between $R$ and $R_{\mathrm{s}}$ has been retained in the proposed model, however, because it might be nonnegligible in regions of very pronounced change in curvature.

\section{Model Validation}

\subsection{Asymptotic Case of Mildly Curved Flow}

[40] Numerous models for velocity (re)distribution in mildly curved open-channel bends have been proposed in literature, e.g., van Bendegom [1947], Rozovskii [1957], Engelund [1974], Odgaard [1981b], Ikeda et al. [1981], Johannesson and Parker [1989b], Seminara and Tubino [1992], Imran et al. [1999], and Camporeale et al. [2007]. Equations (36) and (37) can be simplified for mildly curved flow by assuming that $B / R$ is small, $\psi \approx 1$ and $\partial R_{\mathrm{s}}^{-1} / \partial \mathrm{s} \approx$ $\partial R^{-1} / \partial s$ [equation (40)] leading to:

$$
\lambda_{\alpha \mathrm{s} / \mathrm{R}}(\text { weak curvature })=\frac{1}{2} \frac{H}{C_{\mathrm{f}}} \quad \text { and }
$$

$$
\begin{aligned}
F_{\alpha \mathrm{s} / \mathrm{R}}(\text { weak curvature })= & \frac{1}{2} \frac{S_{\mathrm{n}} F r^{2}+A_{0}-1}{R}-\frac{1}{2} \frac{H}{C_{\mathrm{f}}} \frac{\partial}{\partial s}\left(\frac{1}{R}\right) \\
& +\frac{4 \chi}{C_{\mathrm{f}}} \frac{H^{2}}{B^{2}} \frac{\left\langle f_{\mathrm{s}} f_{\mathrm{n}}\right\rangle_{0}}{R} .
\end{aligned}
$$

The subscript " 0 " denotes values obtained by a model for mildly curved flow. When taking $\chi=1.5$ [equation (A21)], equations (41) and (42) are identical to the model developed by Johannesson and Parker [1989b] based on a smallperturbation approach. It can be concluded that the proposed model without curvature limitations encompasses existing mild curvature models.

\subsection{Asymptotic Case of Straight Flow}

[41] In straight flow, the ratios $1 / R$ and $B / R$ are 0 and $\psi=1$. According to their definitions [equations (25) and (26)], the ratios $\alpha_{\mathrm{S}} / R$ and $A / R$ parameterize adequately the transverse velocity distribution and bed slope in straight rivers. Curvature-induced secondary flow cells may exist in a straight river reach, e.g., as a remnant cells generated in an upstream bends. Their effect is represented by the ratio $\left\langle f_{\mathrm{s}} f_{\mathrm{n}}\right\rangle /$ $R$ [equation (13)], which remains valid in straight flow. In straight flow, equations (36) and (37) reduce to

$$
\begin{gathered}
\lambda_{\alpha \mathrm{s} / R}(\text { straight })=\frac{1}{2} \frac{H}{C_{\mathrm{f}}} \text { and } \\
\mathrm{F}_{\alpha \mathrm{s} / R}(\text { straigth })=\frac{1}{2} \frac{A}{R}+\frac{6}{C_{\mathrm{f}}} \frac{H^{2}}{B^{2}} \frac{\left\langle f_{\mathrm{s}} f_{\mathrm{n}}\right\rangle}{R} .
\end{gathered}
$$

In the absence of secondary flow, the velocity redistribution in a straight river is governed by the bed topography. In a straight river without streamwise variations in bed topogra- 


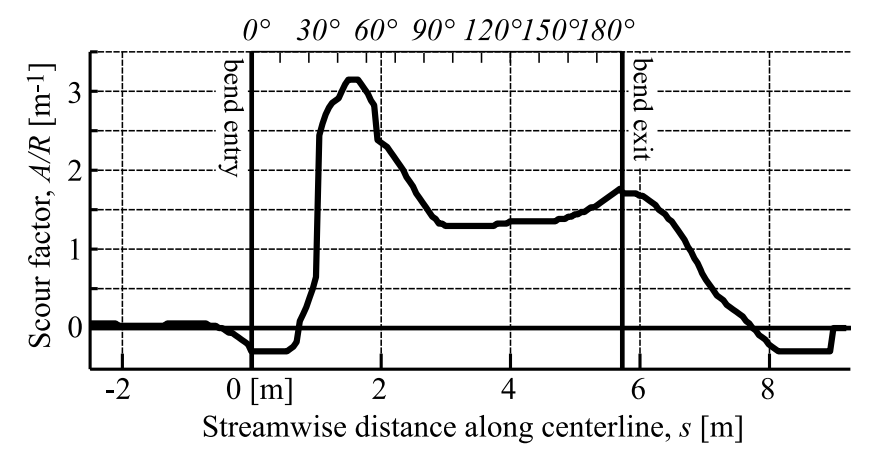

Figure 3. Scour factor $A / R$ [equation (25)] estimated from the measured morphology in the M_16_90_00 experiment over mobile bed.

phy, the model reduces to $\alpha_{\mathrm{s}}=A / 2$, which is equivalent to the traditional Chézy formula that expresses that $U_{\mathrm{s}} \sim \sqrt{\mathrm{h}}$.

\subsection{Flow in Sharply Curved Open-Channel Bends over Horizontal and Mobile Bed Morphologies}

[42] Model predictions for the velocity redistribution, the transverse bed shear stress induced by the secondary flow and curvature-induced energy losses will now be analyzed and compared to experimental data from laboratory experiments in an extremely sharply curved laboratory flume. The flume is $1.3 \mathrm{~m}$ wide and consists of a $193^{\circ}$ bend with a constant centerline radius of curvature of $R=1.7 \mathrm{~m}$, preceded and followed by straight reaches 9 and $5 \mathrm{~m}$ long, respectively. The vertical banks are made of PVC. The bed is covered by a quasiuniform sand with a diameter $d$ of $0.002 \mathrm{~m}$. In the F_16_90_00 experiment over the horizontal bed, the sand bed was frozen by means of a paint sprayed on it, thus preserving the sand roughness. In the M_16 90 00 experiment, a sediment discharge of $0.023 \mathrm{~kg} / \mathrm{m} / \mathrm{s}$ was fed at the flume inlet, leading to the development of a pronounced bar-pool morphology that is typical of open-channel bends. Blanckaert [2010, Figure 6] reports in detail this morphology, which is parameterized in the proposed model by means of the scour factor $A / R$ [equation (25)] represented in Figure 3. The values are similar to typical values of $A$ in the range of 2.5 to 6 for natural streams [Odgaard, 1981a; Ikeda et al., 1981]. The observed pronounced streamwise morphologic variations are in agreement with theoretical model concepts [de Vriend and Struiksma, 1984; Struiksma et al., 1985; Odgaard, 1986] that predict an evolution toward the equilibrium transverse bed slope in a damped oscillated way, including an overshoot of the equilibrium transverse bed slope in the first part of the bend. Table 2 summarizes the geometric and hydraulic conditions in both experiments. Velocity measurements were made with high spatial resolution in 12/13 cross sections around the flume in the F_16_90_00/M_16_90_00 experiments. Blanckaert [2010] reports detailed information on the experimental setup, the measuring techniques, the measuring grids, the data treatment procedures, and the uncertainty in the experimental data.

[43] The curvature ratio $R / B$ of 1.31 corresponds to very sharp bends as found in natural rivers. With aspect ratios of $B / H$ of 8.2 and 9.2, the cross section in the laboratory flume is narrower than in most natural rivers, which amplifies the effects of the secondary flow [equation (37)] and of the hydrodynamic nonlinearities and causes complex threedimensional flow patterns where the influence of bank boundary layers may be significant. In the horizontal bed experiment, for example, flow separates at the inner bank, whereas a counterrotating outer bank cell of secondary flow occurs at the outer bank [Zeng et al., 2008]. In the mobile bed experiment, flow recirculates over the point bar at the inner bank downstream of the bend entry [Blanckaert, 2010, Figure 13]. Obviously, these complex three-dimensional flow features are not accounted for in the proposed model. Curvature discontinuities occur at the inlet and outlet of the bended reach in the laboratory flume. These discontinuous curvatures, which locally cause considerable gradients in flow variables, are not relevant for natural rivers. Because the proposed model cannot treat discontinuous curvatures, the geometric curvature was approximated by a continuous function by means of the relaxation equation $2 H\left(\partial R_{\text {cont }}^{-1} / \partial \mathrm{s}\right)+$ $R_{\text {cont }}^{-1}=R^{-1}$. The narrow cross section and the discontinuities in curvature make these laboratory experiments extreme cases for model validation where curvature effects are amplified with respect to sharply curved natural rivers.

[44] Figures $4 \mathrm{a}$ and $4 \mathrm{~b}$ compare the evolution of $\alpha_{\mathrm{s}} / R$ around the flume in the horizontal and mobile bed experiments, respectively, obtained from (1) the experimental data, (2) the proposed model without curvature restrictions, and (3) the proposed model in its asymptotic formulation for mild curvature. The depth-averaged flow fields in the F_16_90_00 and M_16_90_00 experiments have been reported in detail by Zeng et al. [2008] and Blanckaert [2010], respectively. In both experiments, the velocities increase/decrease rapidly in the inner/outer half of the cross section just downstream of the bend entry and tend to a potential-vortex distribution characterized by $\alpha_{\mathrm{s}} / R=-1 / R=$ -0.59 . This rapid redistribution can be attributed to the abrupt change in curvature. In the F_16_90_00 experiment over the horizontal bed, the velocities gradually increase/ decrease in the outer/inner half of the cross section. Just downstream of the bend exit, they tend toward a forced-

Table 2. Hydraulic and Geometric Conditions in the Experiments

\begin{tabular}{|c|c|c|c|c|c|c|c|c|c|c|c|c|c|c|}
\hline Label & $\underset{[\mathrm{L} / \mathrm{s}]}{Q}$ & $\begin{array}{c}q_{\mathrm{s}} \\
(\mathrm{kg} / \mathrm{s} / \mathrm{m})\end{array}$ & $\begin{array}{c}\tilde{H} \\
(\mathrm{~m})\end{array}$ & $\begin{array}{c}\tilde{U} \\
(\mathrm{~m} / \mathrm{s})\end{array}$ & $\begin{array}{c}E_{s, 0} \\
\left(10^{-4}\right)\end{array}$ & $\begin{array}{c}C_{\mathrm{f}, 0}^{-1 / 2} \\
(-)\end{array}$ & $\begin{array}{c}\tilde{u}_{*} \\
(\mathrm{~m} / \mathrm{s})\end{array}$ & $\begin{array}{c}\tilde{E}_{s} \\
\left(10^{-14}\right)\end{array}$ & $\begin{array}{c}\tilde{C}_{\mathrm{f}}^{-1 / 2} \\
(-)\end{array}$ & $\begin{array}{c}R e \\
\left(10^{3}\right)\end{array}$ & $\begin{array}{l}F r \\
(-)\end{array}$ & $\begin{array}{c}R / B \\
(-)\end{array}$ & $\begin{array}{c}R / H \\
(-)\end{array}$ & $\begin{array}{c}B / H \\
(-)\end{array}$ \\
\hline F_16_90_00 & 89 & - & 0.159 & 0.43 & 6.2 & 14.7 & 0.029 & 8.5 & 13.2 & 69 & 0.35 & 1.31 & 10.6 & 8.2 \\
\hline M_t $1 \overline{6}+90+00$ & 89 & 0.023 & 0.141 & 0.49 & 21.5 & 14.0 & 0.035 & 26.7 & 8.9 & 68 & 0.41 & 1.31 & 12.1 & 9.2 \\
\hline
\end{tabular}

$Q$ is the flow discharge, $q_{\mathrm{s}}$ is the sediment discharge, $\tilde{H}$ is the flume-averaged flow depth, $\tilde{U}$ is the flume averaged velocity equal to $Q / B \tilde{H}, E_{\mathrm{s}, 0}$ is the average energy slope in straight inflow, $\tilde{C}_{\mathrm{f}, 0}^{-1 / 2}$ is the dimensionless Chézy friction coefficient for the straight inflow based on the hydraulic radius $R_{\mathrm{h}}$ equal to $U_{0} / \sqrt{g R_{h, 0}, E_{\mathrm{s}, 0}}, \tilde{u}_{*}$ is the equivalent straight flow shear velocity equal to $C_{\mathrm{f}, 0}^{1 / 2} U, \tilde{E}_{\mathrm{s}}$ is the flume-averaged energy slope, $\tilde{C}_{\mathrm{f}}^{-1 / 2}$ is the dimensionless Chézy friction coefficient based on flume-averaged flow characteristics equal to $\tilde{U} / \sqrt{g \tilde{R}_{h}, \tilde{E}_{\mathrm{s}}}, \operatorname{Re}$ is the Reynolds number equal to $\tilde{U} \tilde{H} / \nu, F r$ is the Froude number equal to $\tilde{U} / \sqrt{g \tilde{H}}$, and $B$ is the flume width. Notations will be simplified henceforward by dropping the tildes on flume-averaged values. 

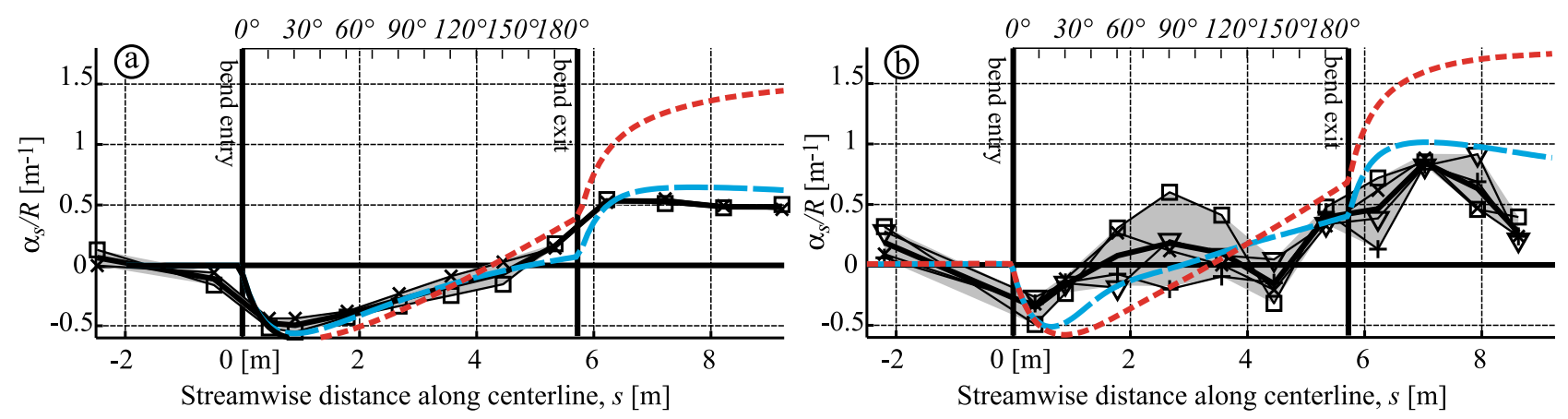

Figure 4. Evolution of $\alpha_{\mathrm{s}} / R$ around the flume in (a) the F_16_90_00 experiment with horizontal bed and (b) the M_16_90_00 experiment with mobile bed morphology obtained from the following: (1) the experimental data by fitting exponential and linear profiles to the width distributions of $U_{\mathrm{s}}$ [equation (26) labeled " $\mathrm{x}$ " and " $\square$," respectively] and $U_{\mathrm{s}} h$ [equation (31), labeled "+" and " $\nabla$," respectively], whereby the full black line represents the average of the different estimations and the gray area indicates their variability; (2) the proposed model without curvature restrictions (long-dashed blue line); and (3) the proposed model in its asymptotic formulation for mild curvature (short-dashed red line).

vortex distribution characterized by $\alpha_{\mathrm{s}} / R=1 / R=0.59$. The gradual outward velocity redistribution can be attributed to the advective momentum transport by the secondary flow. Moreover, the abrupt change in curvature at the bend exit locally contributes significantly to the outward velocity redistribution. In the straight outflow, the velocity distribution hardly varies and remains outward skewed because of the remnant secondary flow cell, which only slowly decays. In the M_16_90_00 experiment, the pronounced mobile bed morphology leaves a strong footprint on the velocity redistribution. The outward increase in flow depth causes a corresponding outward increase in velocity, as indicated by Chézy's law. Moreover, the pronounced streamwise variations in the morphology (Figure 3) cause outward/inward cross flow $U_{\mathrm{n}}$ in regions where the transverse bed slope increases/decreases and the corresponding outward/inward velocity redistribution. Obviously, the complex threedimensional velocity pattern can only be parameterized by means of the transverse profile function with 1 degree of

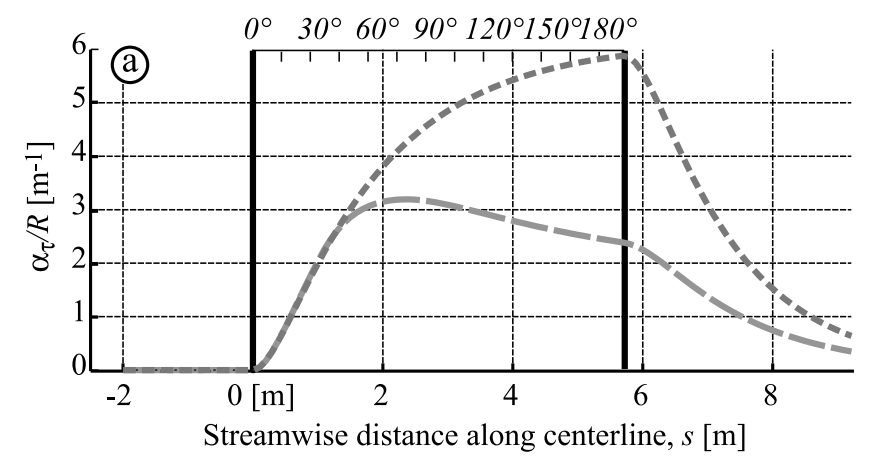

freedom $\alpha_{\mathrm{s}} / R$ in an approximate way. Figure 4 includes estimates of the $\alpha_{\mathrm{s}} / R$ evolution obtained by fitting exponential and linear profiles to the width distributions of $U_{\mathrm{s}}$ [equations (26), labeled " $x$ " and " $\square$," respectively] and $U_{\mathrm{s}} h$ [equation (31), labeled "+" and " $\nabla$," respectively]. The full black line in Figure 4 represents the average of the different estimations, whereas the gray area indicates their variability. A large variability indicates that the transverse velocity distribution deviates considerably from the exponential or linear profile functions adopted in the proposed model. The variability is particularly large in the M_16_90_00 experiment between $60^{\circ}$ and $120^{\circ}$ in the bend and just downstream of the bend exit in regions where flow recirculation occurs near the inner bank [see Blanckaert, 2010]. The mild curvature model significantly overestimates the outward velocity redistribution in both experiments. Although significant deviations occur locally, the proposed model without curvature restrictions agrees globally satisfactorily with the data. The major discrepancies occur in regions where the

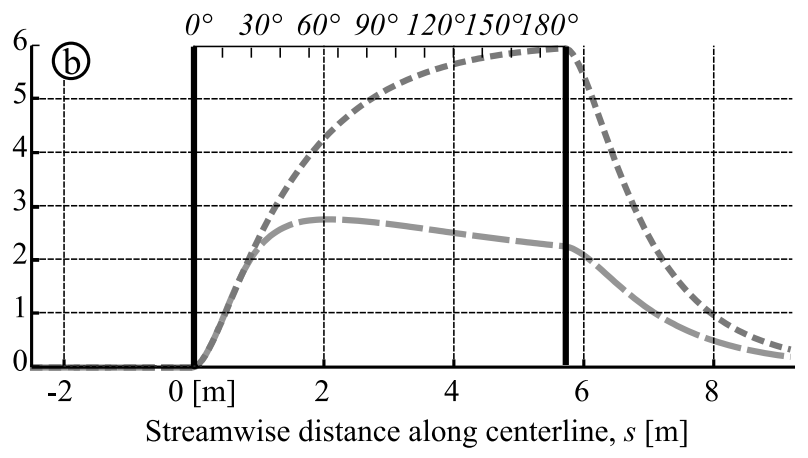

Streamwise distance along centerline, $s[\mathrm{~m}]$

\begin{tabular}{|c|c|c|c|}
\hline \multicolumn{2}{|c|}{ F_16_90_00 } & \multicolumn{2}{c|}{ M_16_90_00 } \\
\hline Mild-curvature model & Model without curvature restrictions & Mild-curvature model & Model without curvature restrictions \\
\hline 3.53 & 1.99 & 3.53 & 1.78 \\
\hline
\end{tabular}

Figure 5. Evolution around the flume of $\alpha_{\tau} / R$ according to equations (14) and (18) (Figure 2) for the model without curvature restrictions (long-dashed blue line) and the mild curvature model (short-dashed red line), respectively, in the (a) F_16_90_00 experiment with horizontal bed and (b) the M_16_90_00 experiment with mobile bed morphology. The average magnitudes of $\alpha_{\tau} / R$ over the flume (excluding the straight inflow reach) are summarized in the table. 
transverse velocity distribution deviates considerably from the exponential or linear profiles adopted in the model. Note that the discontinuity in the forcing centerline curvature is not reflected in the solution for $\alpha_{\mathrm{s}}$ thanks to the degree of freedom associated with the solution of the homogeneous part of equation (35).

[45] Figures 5a and $5 \mathrm{~b}$ compare the evolution of the transverse bed shear stress induced by the secondary flow $\alpha_{\tau} / R$ around the flume in the horizontal and mobile bed experiments, respectively, obtained from the proposed model without curvature restrictions and the proposed model in its asymptotic formulation for mild curvature. The mild curvature model predicts a gradual growth of $\alpha_{\tau} / R$ around the bend followed by a gradual decay in the straight outflow reach, which can both be attributed to inertia [equation (19)]. The model without curvature restrictions, on the opposite, predicts $\alpha_{\tau} / R$ to reach its maximum value at about $60^{\circ}$ in the bend and to decay gradually subsequently. Values at the bend exit are about $25 \%$ lower than the maximum values. This evolution can be attributed to the interaction between the transverse and vertical structures of the flow [equation (18) and Figure 2b]. Averaged over the bend and the straight outflow reach, the overestimations by the mild curvature model are approximately $100 \%$. If coupled to a submodel for the meander morphology (Figure 1), these differences would result in considerable differences in predicted transverse bed slope.

[46] Contrary to the mild curvature model, the proposed model without curvature restrictions gives an estimation of the curvature-induced energy losses [see equations (22) and (23)]. Averaged over the bend reach and the straight outflow, these curvature-induced energy losses are estimated at $21 \%(17 \%$ because of the secondary flow, $1 \%$ because of the nonuniform velocity distribution, and $3 \%$ because of the curvature-induced turbulence) and 30\% (21\% because of the secondary flow, $6 \%$ because of the nonuniform velocity distribution, and $2 \%$ because of the curvature-induced turbulence) in the F_16_90_00 and M_16_90_00 experiments, respectively, which compares favorably with estimated values of about $35 \%$ and $25 \%$ based on the experimental data (Table 2).

[47] To understand differences between the proposed model without curvature restrictions and the mild curvature model, the mechanisms that drive the velocity redistribution [see equations (37) and (42)] are shown in Figures $5 a$ and 5b, which include a table that summarizes the average magnitude of these mechanisms over the flume (excluding the straight inflow reach). A more general parametric analysis of the relevance of the different mechanism will be given in section 5. According to the model, all four mechanisms globally favor outward velocity redistribution and are of dominant order of magnitude in the experiments, although the fourth term is obviously negligible in the horizontal bed experiment. Remarkably, variations in curvature (term II) contribute most to the velocity redistribution in both experiments. Term II reaches high values just downstream of the curvature discontinuities and is negligible in the constant-curvature bend because of the artificial planform of the laboratory flume. Its flume-averaged value, however, is representative of sharp bends in natural rivers with gradually varying curvature. The mild curvature model overestimates the secondary flow effect (term III) by about $250 \%$ and falsely indicates it as the dominant mechanism for velocity redistribution. Nonlinear interactions between the vertical and transverse structures of the flow [equation (18) and Figure 2b] cause the secondary flow effects to decrease considerably in the second part of the bend. Streamwise changes in the morphology and water surface topography (term IV), which are not accounted for in the mild curvature model, yield the smallest contribution to the velocity redistribution, although it is still of dominant order of magnitude. This is the only term that alternates between positive and negative values around the bend. The aforementioned curvature-induced increase in energy losses of about $21 \%$ and $30 \%$ in the flat and mobile bed experiments, respectively, causes a similar reduction on the driving mechanism for the velocity redistribution, as indicated by the factor $1 / \psi$ that appears in terms II, III, and IV. Globally, the mild curvature model overestimates the driving mechanisms for the velocity redistribution by about $50 \%$.

\section{Model Interpretation}

[48] The proposed model [equations (35) to (37)] is relatively simple, transparent, and insightful. The order of magnitude of the different terms in equations (36) and (37) can be estimated as:

$$
\begin{gathered}
O\left(\lambda_{\alpha \mathrm{s} / \mathrm{R}}\right)=\frac{1}{2} \frac{H}{\psi C_{\mathrm{f}}}\left[1-O\left(\frac{\alpha_{\mathrm{s}}}{10} \frac{B^{2}}{R^{2}}\right)\right] \text { and } \\
O\left(F_{\alpha \mathrm{s} / R}\right)=\frac{1}{\mathrm{R}}\left\{O\left(\frac{A-1}{2}\right)-O\left(\frac{1}{2} \frac{H}{C_{\mathrm{f}} R} \frac{R}{\lambda_{\mathrm{A}}}\right)\right. \\
\left.+O\left[\frac{2}{C_{\mathrm{f}}} \frac{H^{2}}{B^{2}}\left(1+\frac{1}{2} \frac{B^{2}}{R^{2}}\right)\right]+O\left(\frac{A}{24} \frac{H}{C_{\mathrm{f}} R} \frac{B^{2}}{R^{2}} \frac{R}{\lambda_{\mathrm{A}}}\right)\right\} .
\end{gathered}
$$

A reasonable upper bound for $\alpha_{\mathrm{s}}$ is $2 R / B$, which corresponds to a linear velocity distribution that decreases from $2 U$ near the outer bank to 0 near the inner bank and which could be a first approximation of the onset of flow separation at the inner bank. The deviation of the adaptation length of the velocity redistribution $\lambda_{\alpha \mathrm{s} / R}$ from its mild curvature approximation [equation (45) versus equation (41)] is only nonnegligible for very sharp bends. Equations (37) and (46) clearly identify the processes that drive the velocity redistribution as well as their parametrical dependence:

[49] 1. The first term, representing the influence of the transverse bed and water surface slopes, is never negligible.

[50] 2. The importance of the second term, representing the influence of changes in curvature, increases with the parameter $C_{\mathrm{f}}^{-1} H / R$. It is negligible in mildly curved bend but of leading order of magnitude if $C_{\mathrm{f}}^{-1} H / R=\mathrm{O}(1)$, which is the case in moderately and sharply curved bends.

[51] 3. The third term, representing advective velocity redistribution by the secondary flow, scales with the square of the aspect ratio. It is of leading order of magnitude for narrow rivers, $B / H<10$, and negligible for very shallow ones, $B / H>50$, indicating important differences between the hydrodynamic mechanisms in shallow natural rivers and in commonly used narrow laboratory flumes.

[52] 4. The last term indicates the influence of streamwise variations in the transverse bed and water surface slopes. The term increases with the parameter $C_{\mathrm{f}}^{-1} H / R$ but remains typically considerably smaller than the second term. This mechanism is not accounted for in mild curvature models [equation (42)]. 

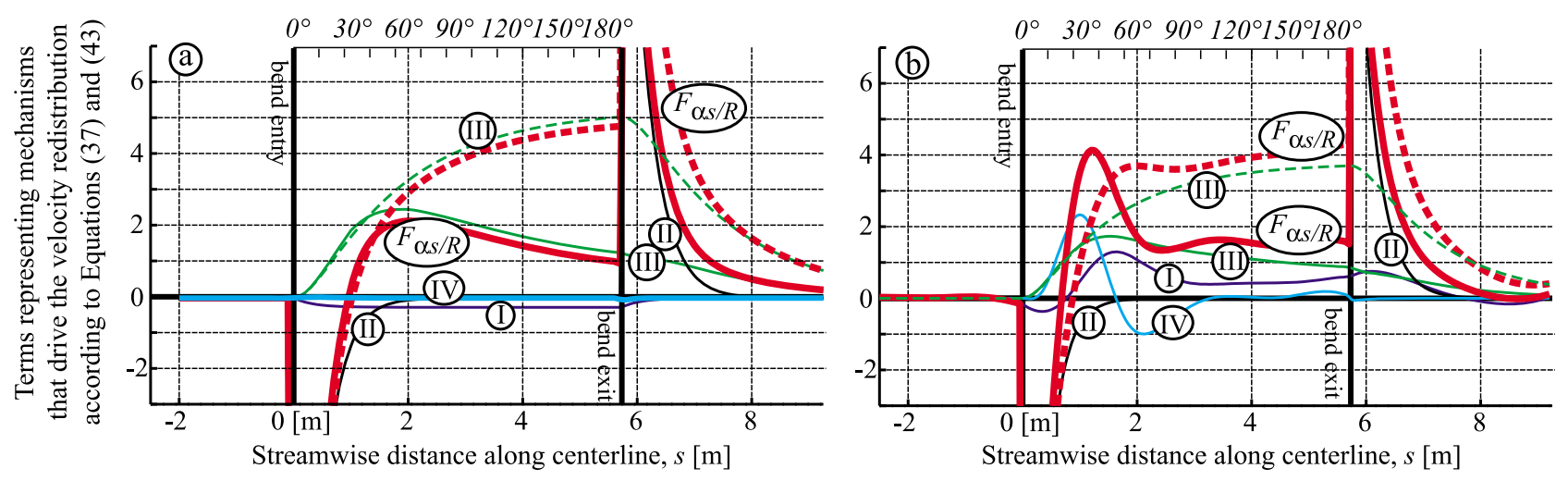

\begin{tabular}{|l|l|c|c|c|c|}
\hline & & \multicolumn{2}{|c|}{ F_16 90_00 } & \multicolumn{2}{c|}{ M_16_90_00 } \\
\hline $\begin{array}{l}\text { Eq. (37) } \\
\text { and (43) }\end{array}$ & \multicolumn{1}{|c|}{ Physical effect } & $\begin{array}{c}\text { Mild-curvature } \\
\text { model }\end{array}$ & $\begin{array}{c}\text { Model without } \\
\text { curvature restrictions }\end{array}$ & $\begin{array}{c}\text { Mild-curvature } \\
\text { model }\end{array}$ & $\begin{array}{c}\text { Model without } \\
\text { curvature restrictions }\end{array}$ \\
\hline Term I & $\begin{array}{l}\text { Transverse bed and } \\
\text { water surface slopes }\end{array}$ & 0.15 & 0.15 & 0.47 & 0.47 \\
\hline Term II & $\begin{array}{l}\text { Streamwise variations } \\
\text { in curvature }\end{array}$ & 1.81 & 1.48 & 1.70 & 1.33 \\
\hline Term III & $\begin{array}{l}\text { Velocity redistribution } \\
\text { by secondary flow }\end{array}$ & 2.94 & 1.24 & 2.29 & 0.86 \\
\hline Term IV & $\begin{array}{l}\text { Streamwise variations in } \\
\text { transverse bed and water } \\
\text { surface slopes }\end{array}$ & - & 0.00 & - & 0.32 \\
\hline$F_{\alpha s R}$ & Sum of physical effects & 4.89 & 2.87 & 4.46 & 2.99 \\
\hline
\end{tabular}

Figure 6. Evolution around the flume of the mechanisms that drive the velocity redistribution according to equations (37) and (42) for the model without curvature restrictions (full lines) and the mild curvature model (dashed lines), respectively, in the (a) F_16_90_00 experiment with horizontal bed and (b) the M_16_90_00 experiment with mobile bed morphology. The labels on the curves correspond to the terms in equations (37) and (42). The average magnitudes of the different mechanisms over the flume (excluding the straight inflow reach) are summarized in the table.

[53] The proposed model identifies $C_{\mathrm{f}}^{-1} H / R$ as the main control parameter with respect to the velocity redistribution in curved open-channel flow. The driving mechanisms related to streamwise variations in curvature and the transverse bed and water surface slopes scale with it and the normalized adaptation length, $\lambda_{\alpha \mathrm{s} / R} / R$ is proportional to it. de Vriend [1981] identified $C_{\mathrm{f}}^{-1} H / R$ as an important control parameter with respect to the formation of an outer-bank cell of secondary flow. Because it represents a ratio between forcing by curvature $(H / R)$ and dissipation by boundary-friction generated turbulence $\left(C_{\mathrm{f}}\right)$, he called $C_{\mathrm{f}}^{-1} H / R$ the Dean number, similar to its definition in curved laminar flow. Blanckaert and de Vriend [2003] identified $C_{\mathrm{f}}^{-1} H / R$ as a major control parameter with respect to the vertical structure of the flow field and its interaction with the transverse flow structure. Johannesson and Parker [1989b] identified a similar parameter, $2 \pi C_{\mathrm{f}}^{-1} H / \lambda_{\mathrm{m}}\left(\lambda_{\mathrm{m}}\right.$ is the meander wavelength), which they called the reduced wave number. Bolla Pittaluga et al. [2009] identified the rather similar parameter $C_{\mathrm{f}}^{-1 / 2} H / R$ as the scaling parameter for curvature (Table 1). According to Johannesson and Parker [1989b] and Imran et al. [1999], the secondary flow scales with $C_{\mathrm{f}}^{-1} 2 H / B$ (Table 1), which can be written as $\left(C_{\mathrm{f}}^{-1} H / R\right)(2 R / B)$. The ratio $B / R$ is traditionally the major scaling parameter used in field studies on meandering rivers. According to its definition, $B / R$ is a major parameter with respect to the velocity excess at the outer bank, which can be written as $\Delta U_{\mathrm{s}} / U=\alpha_{\mathrm{s}}(B / 2 R)$ when a linear width distribution of the velocity is adopted [equation (26)]. With respect to the velocity redistribution, $B / R$ does not play a major role in mildly curved bends [see equations (41) and (42)]. In sharp bends, however, it plays an important role as indicated by the sharp curvature terms in equations (35) to (37) that scale with $B / R$.

[54] The order-of-magnitude analysis indicates fundamental differences between the hydrodynamic mechanisms in shallow natural rivers and in commonly used laboratory flumes with narrow cross section, horizontal bed, and often smooth boundaries (Blanckaert and Graf's [2001] Table 1 summarizes reported laboratory experiments). Advective velocity redistribution by the secondary flow is negligible in the former configuration and dominant in the latter one. This does not mean, however, that secondary flow is not relevant in shallow and mildly curved natural river bends, where the velocity distribution is mainly conditioned by the transverse bed slope [equation (42)], which itself is strongly conditioned by the transverse component of the bed shear stress induced by the secondary flow [see equations (2) and (3)].

[55] According to the order-of-magnitude analysis [equations (45) and (46)], the major sharp curvature effects in natural rivers are due to the following:

[56] 1 . The coupling between the transverse and vertical structures of the flow field, which is apparent in the pro- 
posed model through the mutual dependence of $\alpha_{\mathrm{s}} / R, \alpha_{\tau} / R$, $\left\langle f_{\mathrm{s}} f_{\mathrm{n}}\right\rangle / R$, and $\psi$ [equations (17), (18), and (37) and Figure 1]. It causes a reduction of the secondary flow and its induced transverse component of the bed shear stress, leading to a reduction of the transverse bed slope [see equations (2), (3), (14), and (18)]. The latter always exerts an influence of dominant order of magnitude on the velocity redistribution [term I in equation (37)]. Moreover, it also reduces the velocity redistribution by the secondary flow, although this mechanism is only relevant in relatively narrow rivers [term III in equation (37)]. Finally, the deformation of the streamwise velocity profile and the secondary flow generate additional energy losses parameterized by $\psi$ that directly affect the driving mechanisms for the velocity redistribution [terms II, III, and IV in equation (37)]. These sharp curvature effects are not accounted for in mild curvature models that prescribe a vertical flow structure that is independent of the transverse one (Figure 1) and neglect curvature-induced energy losses.

[57] 2. Mechanisms represented by additional terms in the model [equations (36) and (37) versus equations (41) and (42)], which scale with $B / R$ and are therefore only relevant in sharp natural river bends. Term IV in equation (37), mainly representing velocity redistribution induced by streamwise morphologic variations, is expected to be the principal additional sharp curvature term.

[58] The relevance of these high-curvature effects has been illustrated by means of the laboratory experiments shown in Figures 4, 5, and 6.

\section{Conclusions}

[59] Despite the rapid evolution of computational power, simulation of meandering river flow, morphology, and planform by means of reduced and computationally less expensive models will remain practically relevant for the investigation of large-scale and long-term processes, probabilistic predictions, or rapid assessments. Existing meander models are invariantly based on the assumption of mild curvature and slow curvature variations and fail to explain or reproduce meander dynamics in the high-curvature range. This article proposed a model for meander hydrodynamics without curvature restrictions, which is computationally hardly more expensive than existing mild curvature models. The main originality and contribution of the proposed model is the detailed modeling of the vertical structure of the flow, and especially of the secondary flow and its induced advective momentum transport and transverse component of the bed shear stress. The latter was identified by Camporeale et $a l$. [2007] as the major and most sensitive feedback mechanism between the flow and the morphology in meanders.

[60] The proposed model is based on the development and modeling of a transport equation for the transverse gradient of the velocity, parameterized by $\alpha_{\mathrm{s}} / R$ [equation (26)]. Blanckaert and de Vriend [2003] already identified $\alpha_{\mathrm{s}} / R$ as the principal control parameter for the interaction between the vertical and transverse structures of the flow. Besides the transverse flow structure, the model also provides the magnitude of the secondary flow, the direction of the bed shear stress, and the curvature-induced additional energy losses.

[61] The proposed model has been validated by means of theoretical asymptotic cases and experimental data: it encompasses existing mild curvature models, remains valid for straight flow, and agrees satisfactorily with experimental data from laboratory experiments under conditions that are more demanding than even the sharpest natural river meanders.

[62] The model clearly reveals the mechanisms that drive the velocity redistribution in meander bends as well as their dependence on geometric and hydraulic parameters including the river's roughness $C_{\mathrm{f}}$, the flow depth $H$, the centerline radius of curvature $R$, the width $B$, and morphologic variations. The proposed model identified $C_{\mathrm{f}}^{-1} H / R$ as the major control parameter with respect to meander hydrodynamics in general, whereas the relative curvature $R / B$ parameterizes high-curvature effects. Both parameters are small in mildly curved bends but $O(1)$ in sharply curved bends, resulting in significant differences in the flow dynamics. Whereas streamwise curvature variations are negligible in mildly curved bends, they are the major mechanisms with respect to the velocity redistribution in very sharp bends. Nonlinear feedback between the vertical and transverse flow structures also plays a dominant role in the sharp meander bends: it increases energy losses and reduces the secondary flow, causing a reduction in transverse bed slope and in velocity redistribution by the secondary flow.

[63] The proposed model enhances the application range of meander models. Moreover, it will allow gain of insight in the hydrodynamics and morphodynamics of meander bends. It will, for example, allow to investigate meander dynamics in the high-curvature range or to analyze the relevance of laboratory experiments for natural river meanders that are typically much rougher and wider and have typically a more pronounced morphology.

\section{Appendix A: Development of the Transport Equation for $\alpha_{\mathrm{s}} /[(1+n / R) R]$ from the Depth- Averaged Continuity and Momentum Equations}

\section{A1. Neglecting Small Terms in the Depth-Averaged Momentum Equations and Modeling Terms Related to the Vertical Structure of the Flow Field According to Blanckaert and de Vriend's [2003] Model}

[64] The term $\partial\left(\left\langle v_{\mathrm{s}}{ }^{*} v_{\mathrm{n}}{ }^{*}\right\rangle h\right) / \partial s$ related to the deformation of the vertical profile of the streamwise velocity and the horizontal turbulent diffusion terms are neglected, reducing the streamwise depth-averaged momentum equation, equation (7), into:

$$
\begin{aligned}
\frac{1}{1+n / R} U_{\mathrm{s}} \frac{\partial U_{\mathrm{s}}}{\partial s} & +U_{\mathrm{s}} U_{\mathrm{n}} \frac{\alpha_{\mathrm{s}}+1}{(1+n / R) R} \approx-\frac{1}{1+n / R} g \frac{\partial z_{\mathrm{s}}}{\partial s} \\
& -\frac{\tau_{\mathrm{bs}}}{\rho h}-\frac{\partial\left\langle v_{\mathrm{s}}^{*} v_{\mathrm{n}}^{*}\right\rangle}{\partial n}-\left\langle v_{\mathrm{s}}^{*} v_{\mathrm{n}}^{*}\right\rangle\left[\frac{1}{h} \frac{\partial h}{\partial n}+\frac{2}{(1+n / R) R}\right],
\end{aligned}
$$

where $\left\langle v_{\mathrm{s}}^{*} v_{\mathrm{n}}^{*}\right\rangle$ represents transport of streamwise flow momentum by the secondary flow in transverse direction. Existing analytical models for secondary flow [e.g., van Bendegom, 1947; Rozovskii, 1957; Engelund, 1974; de Vriend, 1977; Johannesson and Parker, 1989a] describe this term in the central portion of wide open-channel bends according to equation (13). The term $\left\langle v_{\mathrm{s}}^{*} v_{\mathrm{n}}^{*}\right\rangle$ can reasonably 
be expected to reach its maximum values in the center of the secondary flow cell, situated at $\mathrm{n}=\mathrm{nc}$, where

$$
\left\langle v_{\mathrm{s}}^{*} v_{\mathrm{n}}^{*}\right\rangle_{\mathrm{nc}}=\left[U_{\mathrm{s}}^{2} \frac{h\left\langle f_{\mathrm{s}} f_{\mathrm{n}}\right\rangle}{(1+n / R) R}\right]_{\mathrm{nc}} .
$$

The distribution of the term $\left\langle v_{\mathrm{s}}^{*} v_{\mathrm{n}}^{*}\right\rangle$ over the entire width of the river will now be defined as:

$$
\left\langle v_{\mathrm{s}}^{*} v_{\mathrm{n}}^{*}\right\rangle=\left\langle v_{\mathrm{s}}^{*} v_{\mathrm{n}}^{*}\right\rangle_{\mathrm{nc}} g_{\mathrm{sn}}(n) \approx \frac{U^{2} H\left\langle f_{\mathrm{s}} f_{\mathrm{n}}\right\rangle}{R} g_{\mathrm{sn}}(n),
$$

where $U$ and $H$ are cross-sectional averaged values and $R$ is the radius of curvature at the river centerline. The transverse profile $g_{\mathrm{sn}}(n)$ is 0 at the river banks that are impermeable for momentum and reaches its maximum values of $g_{\mathrm{sn}}(\mathrm{nc})=\chi=$ $O(1)$ in the eye of the secondary flow cell. The streamwise component of the bed shear stress in equation (5) is modeled as follows:

$$
\begin{aligned}
\frac{\tau_{\mathrm{bs}}}{\rho} & =\psi C_{\mathrm{f} 0} \sqrt{U_{\mathrm{s}}^{2}+U_{\mathrm{n}}^{2}} U_{\mathrm{s}} \approx \psi C_{\mathrm{f} 0} U_{\mathrm{s}}^{2} \\
& =\left(\psi_{\text {secondary flow }} \psi_{\alpha_{\mathrm{s}}} \psi_{\text {turbulence }}\right) C_{\mathrm{f} 0} U_{\mathrm{s}}^{2},
\end{aligned}
$$

where the amplification factor $\psi$ accounts for curvatureinduced increases in bed shear stress and energy losses [section 2; compare equations (21), (22), and (23)].

\section{A2. Development of the Transport Equation for $\alpha_{\mathrm{s}} /[(1+n / R) R]=\left(\partial U_{\mathrm{s}} / \partial n\right) / U_{\mathrm{s}}$}

[65] When substituting the term $\partial U_{\mathrm{S}} / \partial s$ according to equation (A1), expressing the term $\left\langle v_{\mathrm{s}}^{*} v_{\mathrm{n}}^{*}\right\rangle$ according to equation (A3), and the term $\tau_{\mathrm{bs}} / \rho$ according to equation (A4), the first term in the second line of equation (29) can be developed as shown in equation (A5). Development of the derivatives and substitution of the definition of $\alpha_{\mathrm{s}} /[(1+n / R) R]$ [equation (15)] yields, after some tedious but straightforward mathematics, equation (A6). The second term in the second line of equation (29) can be developed in a similar way, as shown in equation (A7). Summation of the right-hand side in equations (A6) and (A7) gives the right-hand side in equation (30).

\section{A3. Modeling the Water Surface Elevation $z_{\mathrm{s}}$ and Its Gradients}

[66] The water surface elevation is approximated by the sum of the water surface elevation at the centerline and a curvature-induced transverse tilting (superelevation) that is constant over the width of the river:

$$
z_{s}(s, n)=z_{s}(s, 0)+n \frac{\partial z_{s}}{\partial n}(s)
$$

A change in the transversal tilting of the water surface induced by a change in geometric river curvature requires transverse mass transport. As a result, the transverse tilting of the water surface lags behind the geometric river curvature. The transverse mass transport and the spatial lag can be accounted for by considering the depth-averaged transverse momentum equation [Blanckaert and de Vriend, 2003]:

$$
\begin{aligned}
\frac{1}{1+n / R} & U_{s} \frac{\partial U_{n}}{\partial s}+U_{n} \frac{\partial U_{n}}{\partial n}-\frac{U_{s}^{2}}{(1+n / R) R}= \\
& -g \frac{\partial z_{s}}{\partial n}-\frac{\tau_{b n}}{\rho h}-\frac{1}{1+n / R} \frac{1}{h} \frac{\partial\left\langle v_{s}^{*} v_{n}^{*}\right\rangle h}{\partial s}-\frac{1}{h} \frac{\partial\left\langle v_{n}^{*} v_{n}^{*}\right\rangle h}{\partial n} \\
& +\frac{\left\langle v_{s}^{*} v_{s}^{*}\right\rangle-\left\langle v_{n}^{*} v_{n}^{*}\right\rangle}{(1+n / R) R}+\mathrm{HDT}_{n} .
\end{aligned}
$$

Writing this equation in a curvilinear reference system $\left(s^{\prime}, n^{\prime}\right)$ that follows the streamlines and where $U_{\mathrm{n}}^{\prime}$ is 0 by definition, and retaining only the two dominant terms yields:

$$
-\frac{U_{s^{\prime}}^{2}}{\left(1+n / R_{S}\right) R_{\mathrm{s}}}=-g \frac{\partial z_{s}}{\partial n^{\prime}} \approx-g \frac{\partial z_{\mathrm{s}}}{\partial n},
$$

where $R_{\mathrm{s}}$ represents the streamline curvature at the centerline. To comply with the assumption that the transverse tilting of the water surface slope is constant, this equation is approximated by:

$$
\frac{\partial z_{s}}{\partial n} \approx S_{n} F r^{2} \frac{H}{R_{s}},
$$

where $F r$ is the Froude number based on the cross-sectional averaged values and defined as $U /(g H)^{1 / 2}$ and $S_{\mathrm{n}}$ is equal

$$
\begin{aligned}
\frac{1}{U_{\mathrm{s}}} \frac{\partial^{2} U_{\mathrm{s}}}{\partial s \partial n}=\frac{1}{U_{\mathrm{s}}} \frac{\partial}{\partial n}\left(\frac{\partial U_{\mathrm{s}}}{\partial s}\right)= & \frac{1}{U_{\mathrm{s}}} \frac{\partial}{\partial n}\left\{-U_{\mathrm{n}} \frac{\alpha_{\mathrm{s}}+1}{R}-\frac{g}{U_{\mathrm{s}}} \frac{\partial z_{\mathrm{s}}}{\partial s}-(1+n / R) \psi C_{\mathrm{f}} \frac{U_{\mathrm{s}}}{h}-\frac{1+n / R}{U_{\mathrm{s}}}\left[\frac{\partial}{\partial n}\left(\frac{U^{2} H\left\langle f_{\mathrm{s}} f_{\mathrm{n}}\right\rangle}{R} g_{\mathrm{sn}}\right)\right.\right. \\
& \left.\left.+\left(\frac{U^{2} H\left\langle f_{\mathrm{s}} f_{\mathrm{n}}\right\rangle}{R} g_{\mathrm{sn}}\right)\left(\frac{1}{h} \frac{\partial h}{\partial n}+\frac{2}{(1+n / R) R}\right)\right]\right\} .
\end{aligned}
$$

$$
\begin{aligned}
\frac{1}{U_{\mathrm{s}}} \frac{\partial^{2} U_{\mathrm{s}}}{\partial s \partial n}= & -\frac{1}{U_{\mathrm{s}}} \frac{\partial}{\partial n}\left(U_{\mathrm{n}} \frac{\alpha_{\mathrm{s}}+1}{R}\right)-\frac{g}{U_{\mathrm{s}}^{2}} \frac{\partial^{2} z_{\mathrm{s}}}{\partial s \partial n}+\frac{\alpha_{\mathrm{s}}}{(1+n / R) R} \frac{g}{U_{\mathrm{s}}^{2}} \frac{\partial z_{\mathrm{s}}}{\partial s}-(1+n / R) \frac{\psi C_{\mathrm{f}}}{h}\left(\frac{\alpha_{\mathrm{s}}+1}{(1+n / R) R}-\frac{1}{h} \frac{\partial h}{\partial n}\right) \\
& -\frac{U^{2} H\left\langle f_{\mathrm{s}} f_{\mathrm{n}}\right\rangle}{U_{\mathrm{s}}^{2} R}\left\{\frac{1-\alpha_{\mathrm{s}}}{R} g_{\mathrm{sn}}\left(\frac{1}{g_{\mathrm{sn}}} \frac{\partial g_{\mathrm{sn}}}{\partial n}+\frac{1}{h} \frac{\partial h}{\partial n}+\frac{2}{(1+n / R) R}\right)+(1+n / R) \frac{\partial}{\partial n}\left[g_{\mathrm{sn}}\left(\frac{1}{g_{\mathrm{sn}}} \frac{\partial g_{\mathrm{sn}}}{\partial n}+\frac{1}{h} \frac{\partial h}{\partial n}+\frac{2}{(1+n / R) R}\right)\right]\right\} .
\end{aligned}
$$

$$
\begin{aligned}
-\frac{\alpha_{s}}{(1+n / R) R} \frac{1}{U_{s}} \frac{\partial U_{s}}{\partial s}= & -\frac{\alpha_{s}}{(1+n / R) R} \frac{1}{U_{s}}\left\{-U_{n} \frac{\alpha_{s}+1}{R}-\frac{g}{U_{s}} \frac{\partial z_{s}}{\partial s}-(1+n / R) \psi C_{f} \frac{U_{s}}{h}-(1+n / R) \frac{U^{2} H\left\langle f_{s} f_{n}\right\rangle}{U_{s} R} g_{s n}\right. \\
& \left.\cdot\left(\frac{1}{g_{s n}} \frac{\partial g_{s n}}{\partial n}+\frac{1}{h} \frac{\partial h}{\partial n}+\frac{2}{(1+n / R) R}\right)\right\}
\end{aligned}
$$


to $O(1)$. Assuming that $S_{\mathrm{n}}, F r$, and $H$ hardly vary in streamwise direction, the streamwise gradient of the transverse tilting of the water surface can be approximated as:

$$
\frac{\partial^{2} z_{s}}{\partial s \partial n}(s)=\frac{\partial}{\partial s}\left(S_{n} F r^{2} \frac{H}{R_{s}}\right) \approx S_{n} F r^{2} H \frac{\partial}{\partial s}\left(\frac{1}{R_{s}}\right) .
$$

Averaged over the cross section, the driving water surface gradient and the resisting boundary friction are in quasiequilibrium, resulting in:

$$
\frac{\partial z_{s}}{\partial s}(s, 0) \approx-\psi C_{f} \frac{U^{2}}{g H} .
$$

The streamwise water surface slope is obtained from equations (A8), (A12), and (A13) as:

$$
\begin{aligned}
\frac{\partial z_{s}}{\partial s}(s, n) & =\frac{\partial z_{s}}{\partial s}(s, 0)+n \frac{\partial^{2} z_{s}}{\partial s \partial n}(s) \\
& =-\psi C_{f} \frac{U^{2}}{g H}+n S_{n} F r^{2} H \frac{\partial}{\partial s}\left(\frac{1}{R_{s}}\right)
\end{aligned}
$$

The terms in the third line of equation (29), representing the effect of the water surface topography on the velocity redistribution, are modeled by substitution of equations (A10) and (A14), resulting in the fourth line in equation (33).

\section{A4. Modeling the Cross Flow, $\boldsymbol{U}_{\mathbf{n}}$}

[67] The unit discharge in transverse direction can be obtained from the depth-averaged continuity equation (4) as:

$$
U_{\mathrm{n}} h=-\frac{1}{1+n / R} \int_{-B / 2}^{n} \frac{\partial U_{\mathrm{s}} h}{\partial s} \mathrm{~d} n .
$$

Modeling $U_{\mathrm{s}}$ and $h$ according to equations (26) and (31), respectively, and assuming that the river width $B$ is constant gives:

$$
\begin{aligned}
U_{\mathrm{n}} h= & -\frac{1}{1+n / R} \frac{\partial}{\partial s}\left\{U_{s}(n=0) h(n=0)\right. \\
& \left.\cdot \int_{-B / 2}^{n}(1+n / R)^{\alpha_{\mathrm{s}}+S_{\mathrm{n}} F r^{2}+A} \mathrm{~d} n\right\} \\
\approx & -\frac{1}{1+n / R} \frac{\partial}{\partial s}\left\{U H \int_{-B / 2}^{n}\left(1+\frac{\alpha_{\mathrm{s}}+S_{\mathrm{n}} F r^{2}+A}{R} n\right) \mathrm{d} n\right\} \\
= & -\frac{U H}{2}\left(n^{2}-\frac{B^{2}}{4}\right) \frac{1}{1+n / R} \frac{\partial}{\partial s}\left(\frac{\alpha_{\mathrm{s}}+S_{\mathrm{n}} F r^{2}+A}{R}\right),
\end{aligned}
$$

which can be further developed in a similar way to give the cross flow:

$$
\begin{aligned}
U_{n}(s, n)= & -\frac{U}{2}\left(n^{2}-\frac{B^{2}}{4}\right)\left(1-\frac{1+S_{n} F r^{2}+A}{R} n\right) \\
& \cdot \frac{\partial}{\partial s}\left(\frac{\alpha_{s}+S_{n} F r^{2}+A}{R}\right) .
\end{aligned}
$$

\section{A5. Averaging Equation (33) over the Width of the River}

[68] Equation (33) is averaged over the width, or more rigorously over an infinitesimal sector of the bend, by means of the following operator that takes into account the metric factor $1+n / R$.

$$
\frac{1}{B} \int_{-B / 2}^{B / 2}(1+n / R)\{\text { equation }(33)\} \mathrm{d} n
$$

The algebraic development of the term-by-term integration makes use of the linearization hypothesis that has been justified in section 2 in the context of equations (25) and (26).

$$
(1+n / R)^{\varepsilon} \approx(1+\varepsilon n / R)
$$

where $\varepsilon$ only varies in streamwise direction. The width averaging of the term in the left-hand side of equation (33) neglects a lower-order contribution:

$$
\begin{gathered}
\frac{1}{B} \int_{-B / 2}^{B / 2}(1+n / R) \frac{\partial}{\partial s}\left[\frac{\alpha_{s}}{(1+n / R) R}\right] \mathrm{d} n=\frac{\partial}{\partial s}\left(\frac{\alpha_{s}}{R}\right)-\alpha_{s} \frac{\partial}{\partial s}\left(\frac{1}{R}\right) \\
\cdot\left(1-\frac{R}{B} \ln \frac{1+B / 2 R}{1-B / 2 R}\right)=\frac{\partial}{\partial s}\left(\frac{\alpha_{s}}{R}\right)\left[1+O\left(\frac{1}{10} \frac{B^{2}}{R^{2}}\right)\right] \\
\approx \frac{\partial}{\partial s}\left(\frac{\alpha_{s}}{R}\right) .
\end{gathered}
$$

The width averaging of the fourth line in equation (33) representing the effect of advective velocity redistribution by the secondary flow requires the definition of the transverse profile $g_{\mathrm{sn}}(\mathrm{n})$. The precise form of this profile is not crucial in the framework of the development of a widthaveraged model, as long as the profile reproduces correctly the zero values at the impermeable riverbanks and the maximum value in the eye of the secondary flow cell. Assuming that this eye of the secondary flow cell is near the centerline, the simplest appropriate formulation of $g_{\mathrm{sn}}(\mathrm{n})$ is defined by a parabolic profile:

$$
g_{\mathrm{sn}}(\mathrm{n})=g_{\mathrm{sn}}(\mathrm{nc})\left[1-\left(\frac{n}{B / 2}\right)^{2}\right]=\chi\left(1-\frac{4 n^{2}}{B^{2}}\right)
$$

where $g_{\mathrm{sn}}(\mathrm{nc})=\chi=O(1)$ as aforementioned.

[69] Acknowledgments. This research was supported by the Swiss National Science Foundation under grants 2100-052257.97 and 2000059392.99 , by the Netherlands Organisation for Scientific Research (NWO) under grant DN66-149, and by the Chinese Academy of Sciences fellowship for young international scientists under grant 2009YA1-2.

\section{References}

Abad, J. D., and M. H. Garcia (2009), Experiments in a high-amplitude Kinoshita meandering channel: 2. Implications of bend orientation on bed morphodynamics, Water Resour. Res., 45, W02402, doi:10.1029/ 2008WR007017. 
Bathurst, J. C., C. R. Thorne, and R. D. Hey (1979), Secondary flow and shear stress at river bends, J. Hydraul. Div. Am. Soc. Civ. Eng., 105(10), 1277-1295.

Blanckaert, K. (2009), Saturation of curvature-induced secondary flow, energy losses, and turbulence in sharp open-channel bends: Laboratory experiments, analysis, and modeling, J. Geophys. Res., 114, F03015, doi:10.1029/2008JF001137.

Blanckaert, K. (2010), Topographic steering, flow recirculation, velocity redistribution and bed topography in sharp meander bends, Water Resour Res., 46, W09506, doi:10.1029/2009WR008303.

Blanckaert, K., and H. J. de Vriend (2003), Nonlinear modeling of mean flow redistribution in curved open channels, Water Resour. Res., 39(12), 1375, doi:10.1029/2003WR002068.

Blanckaert, K., and H. J. de Vriend (2004), Secondary flow in sharp open-channel bends, J. Fluid Mech., 498, 353-380.

Blanckaert, K., and W. H. Graf (2001), Experiments on flow in an openchannel bend. Mean flow and turbulence, J. Hydraul. Eng., 127(10) 835-847.

Blanckaert, K., and W. H. Graf (2004), Momentum transport in sharp open-channel bends, J. Hydraul. Eng., 130(3), 186-198, doi:10.1061/ (ASCE)0733-9429(2004)130:3(186).

Blanckaert, K., I. Schnauder, A. Sukhodolov, W. van Balen, and W. Uijttewaa (2010), Meandering: Field experiments, laboratory experiments and numerical modeling, in River, Coastal and Estuarine Morphodynamics: RCEM 2009, edited by C. A. Vionnet, M. H. Garcia, E. M. Latrubesse, and G. M. E. Perillo, Taylor \& Francis Group, London, ISBN 978-0415-55426-8.

Blondeaux, P., and G. Seminara (1985), A unified bar-theory of river meanders, J. Fluid Mech., 157, 449-470.

Bolla Pittaluga, M., G. Nobile, and G. Seminara (2009), A nonlinear model for river meandering, Water Resour. Res., 45, W04432, doi:10.1029/ 2008WR007298.

Camporeale, C., P. Perona, A. Porporato, and L. Ridolfi (2005), On the long-term behavior of meandering rivers, Water Resour. Res., 41 W12403, doi:10.1029/2005WR004109.

Camporeale, C., P. Perona, A. Porporato, and L. Ridolfi (2007), Hierarchy of models for meandering rivers and related morphodynamic processes, Rev. Geophys., 45, RG1001, doi:10.1029/2005RG000185.

Camporeale, C., E. Perucca, and L. Ridolfi (2008), Significance of cutoff in meandering river dynamics, J. Geophys. Res., 113, F01001, doi:10.1029/ 2006JF000694.

Chang, H. H. (1983), Energy expenditure in curved open channels, J. Hydraul. Eng., 109(7), 1012-1022.

Clevis, Q., G. E. Tucker, G. Lock, S. T. Lancaster, N. Gasparini, A. Desitter, and R. L. Brass (2006a), Geoarchaeological simulation of meandering river deposits and settlement distributions: A three-dimensional approach, Geoarchaeology, 21(8), 843-874.

Clevis, Q., G. E. Tucker, S. T. Lancaster, A. Desitter, N. Gasparini, and G. Lock (2006b), A simple algorithm for the mapping of TIN data on a static grid: Applied to the stratigraphic simulation of river meander deposits, Comput. Geosci., 32(6), 749-766.

Cojan, I., O. Fouche, S. Lopez, and J. Rivoirard (2005), Process-based reservoir modelling in the example of meandering channel, in Quantitative Geology and Geostatistics, edited by O. Leuangthong, and V. C. Deutsch, vol. 14, Part 1-2, 61-619.

Crosato, A. (2008), Analysis and modelling of river meandering, Ph.D. dissertation, Delft Univ. of Technol., Delft, Netherlands.

de Vriend, H. J. (1977), A mathematical model of steady flow in curved shallow channels, J. Hydraul. Res, 15(1), 37-54.

de Vriend, H. J. (1981), Steady flow in shallow channel bends, Rep. 81-3, Fluid Mech. Lab., Dep. of Civ. Eng., Delft Univ. of Technol., Delft, Netherlands.

de Vriend, H. J. and N. Struiksma (1984), Flow and bed deformation in river bends, in River Meandering, edited by C. M. Elliot, pp. 810-828, ASCE, New York.

Duan, J. G., S. S. Y. Wang, and Y. F. Jia (2001), The application of the enhanced CCHE2D model to study the alluvial channel migration process, J. Hydraul. Res., 39(5), 469-480.

Edwards, B. F., and D. H. Smith (2002), River meandering dynamics, Phys. Rev. E, 65, 046303-1-046303-12.

Engelund, F. (1974), Flow and bed topography in channel bends, J. Hydraul. Div. Am. Soc. Civ. Eng., 100(HY11), 1631-1648.

Ferguson, R. I., D. R. Parsons, S. N. Lane, and R. J. Hardy (2003), Flow in meander bends with recirculation at the inner bank, Water Resour. Res. 39(11), 1322, doi:10.1029/2003WR001965.

Fischer-Antze, T., N. R. B. Olsen, and D. Gutknecht (2008), Threedimensional CFD modeling of morphological bed changes in the Danube River, Water Resour. Res., 44, W09422, doi:10.1029/2007WR006402.
Howard, A. D. (1984), Simulation model of meandering, in River Meander ing, edited by C. M. Elliott, pp. 952-963, Am. Soc. Civ. Eng., Reston, Va. Ikeda, S., G. Parker, and K. Sawai (1981), Bend theory of river meanders Part 1: Linear development, J. Fluid Mech., 112, 363-377.

Imran, J., G. Parker, and C. Pirmez (1999), A nonlinear model of flow in meandering submarine and subaerial channels, J. Fluid Mech., 400, 295-331.

Johannesson, H., and G. Parker (1989a), Secondary flow in a mildly sinuous channel, J. Hydraul. Eng., 115(3), 289-308.

Johannesson, H., and G. Parker $(1989 \mathrm{~b})$, Velocity redistribution in meandering rivers, J. Hydraul. Eng. Am. Soc. Civ. Eng., 115(8), 1019-1039.

Khosronejad, A., C. Rennie, S. Neyshabouri, and R. D. Townsend (2007), 3D numerical modeling of flow and sediment transport in laboratory channel bends, J. Hydraul. Eng., 133(10), 1123-1134.

Kikkawa, H., S. Ikeda, and A. Kitagawa (1976), Flow and bed topography in curved open channels, J. Hydraul. Eng. Am. Soc. Civ. Eng., 102(9), 1327-1342.

Lancaster, S. T., and R. L. Bras (2002), A simple model of river meandering and its comparison to natural channels, Hydrol. Processes, 16, 1-26.

Leeder, M. R., and P. H. Bridges (1975), Flow separation in meander bends, Nature, 253(5490), 338-339.

Liverpool, T. B., and S. F. Edwards (1995), Dynamics of a meandering river, Phys. Rev. Lett., 75(16), 3016-3019.

Minh Duc, B., T. Wenka, and W. Rodi (2004), Numerical modelling of bed deformation in laboratory channels, J. Hydraul. Eng., 130(9), 894-904.

Mockmore, C. A. (1943), Flow around bends in stable channels, Trans. Am. Soc. Civ. Eng., 109, 593-628.

Odgaard, A. J. (1981a), Transverse bed slope in alluvial channel bends, J. Hydraul. Eng. Am. Soc. Civ. Eng., 107(HY12), 1677-1694.

Odgaard, A. J. (1981b), Meander flow model. 1: Development, J. Hydraul. Eng. Am. Soc. Civ. Eng., 107(12), 1677-1694.

Odgaard, A. J. (1986), Meander flow model. I: Development, J. Hydraul. Eng., 112(12), 1117-1136.

Odgaard, A. J. (1989), River-meander model. I: Development, J. Hydraul. Eng., 115(11), 1433-1450.

Olesen, K. W. (1987), Bed topography in shallow river bends, Commun. Hydraul. Geotech. Eng., 87-1, Delft Univ. of Technol., Delft, Netherlands. Parker, G., and E. D. Andrews (1986), On the time development of meander bends, J. Fluid Mech., 162, 139-156.

Parker, G., and H. Johannesson (1989), Observations on several recent theories of resonance and overdeepening in meandering channels, in River Meandering, edited by S. Ikeda, and G. Parker, Water Resour. Monogr. $12,379-415$.

Parker, G., K. Sawai, and S. Ikeda (1982), Bend theory of river meanders. Part 2: Nonlinear deformation of finite-amplitude bends, J. Fluid Mech. $115,303-314$

Parker, G., P. Diplas, and J. Akiyama (1983), Meander bends of high amplitude, J. Hydraul. Eng., 109(10), 1323-1337.

Pizzuto, J., and T. Meckelnburg (1989), Evaluation of a linear bank erosion equation, Water Resour. Res., 25, 1005-1013.

Rozovskii, I. L. (1957), Flow of Water in Bends of Open Channels, Acad. Sci. Ukr. SSR, Isr. Progr. Sci. Transl., Jerusalem, 1961.

Ruther, N., and N. R. B. Olsen (2005), 3D modeling of sediment transport in a narrow $90^{\circ}$ channel bend, J. Hydraul. Eng., 131(10), 917-920.

Ruther, N., and N. R. B. Olsen (2007), Modelling free-forming meander evolution in a laboratory channel using three-dimensional computational fluid dynamics, Geomorphology, 89(3-4), 308-319.

Seminara, G. (2006), Meanders, J. Fluid Mech., 554, 271-297.

Seminara, G., and M. Tubino (1992), Weakly nonlinear theory of regular meanders, J. Fluid Mech., 244, 257-288.

Shimizu, Y., H. Yamaguchi, and T. Itakura (1990), Three-dimensional computation of flow and bed deformation, J. Hydraul. Eng., 116(9), 1090-1108.

Stølum, H. H. (1996), River meandering as a self-organization process, Science, 271, 1710-1713.

Stølum, H. H. (1998), Planform geometry and dynamics of meandering rivers, Geol. Soc. Am. Bull., 110(11), 1485-1498.

Struiksma, N., K. W. Olesen, C. Flokstra, and H. J. de Vriend (1985), Bed deformation in curved alluvial channels, J. Hydraul. Res., 23(1), 57-79.

Sun, T., P. Meakin, and T. Jøssang (2001), A computer model for meandering rivers with multiple bed load sediment sizes: 2 . Computer simulations, Water Resour. Res., 37(8), 2243-2258.

Swanson, D. C. (1993), The importance of fluvial processes and related reservoir deposits, J. Pet. Technol., 45(4), 368-377.

van Bendegom, L. (1947), Eenige beschouwingen over riviermorphologie en rivierverbetering (in Dutch), De Ingenieur, 59(4), 1-11. 
van Vuren, S., H. J. de Vriend, S. Ouwekerk, and M. Kok (2005), Stochastic modeling of the impact of flood protection measures along the River Waal in the Netherlands, Nat. Hazards, 36(1-2), 81-102.

Wu, W., W. Rodi, and T. Wenka (2000), 3D numerical modeling of flow and sediment transport in open channels, J. Hydraul. Eng., 126(1), 4-15.

Zeng, J., G. Constantinescu, K. Blanckaert, and L. Weber (2008), Flow and bathymetry in sharp open-channel bends: Experiments and predictions, Water Resour. Res., 44, W09401, doi:10.1029/2007WR006303.
Zolezzi, G., and G. Seminara (2001), Downstream and upstream influence in river meandering. Part 1: General theory and application to overdeepening, J. Fluid Mech., 438, 183-211.

K. Blanckaert, LCH-ENAC, Ecole Polytechnique Fédérale Lausanne, CH-1015 Lausanne, Switzerland. (koen.blanckaert@epfl.ch)

H. J. de Vriend, Department of Civil Engineering and Geosciences, Delft University of Technology, Delft, PO Box 5048, The Netherlands. 